\title{
Supersymmetric anti-D3-brane action in the Kachru-Kallosh-Linde-Trivedi setup
}

\author{
Niccolò Cribiori@,$^{1, *}$ Christoph Roupec, ${ }^{1, \dagger}$ Timm Wrase $\odot,{ }^{1, \hbar}$ and Yusuke Yamada $\odot^{2,3, \S}$ \\ ${ }^{1}$ Institute for Theoretical Physics, TU Wien, Wiedner Hauptstrasse 8-10/136, A-1040 Vienna, Austria \\ ${ }^{2}$ Department of Physics, Graduate School of Science, The University of Tokyo, \\ Hongo 7-3-1 Bunkyo-ku, Tokyo 113-0033, Japan \\ ${ }^{3}$ Research Center for the Early Universe (RESCEU), Graduate School of Science, The University of Tokyo, \\ Hongo 7-3-1 Bunkyo-ku, Tokyo 113-0033, Japan
}

(Received 1 July 2019; published 3 September 2019)

\begin{abstract}
An anti-D3-brane plays a crucial role in the construction of semirealistic cosmological models in string theory. Part of its action provides an uplift term that has been used to lift anti-de Sitter solutions to phenomenologically viable de Sitter vacua in the Kachru-Kallosh-Linde-Trivedi and large volume scenario (LVS) setups. In the last few years it has been shown that this uplift breaks supersymmetry spontaneously and can be described in the four-dimensional $\mathcal{N}=1$ supergravity language by using constrained supermultiplets. Here we derive the complete four-dimensional $\mathcal{N}=1$ supergravity action for an antiD3-brane coupled to all closed-string background fields. In particular, we include the vector field, the scalar fields, and all fermions that live on the anti-D3-brane.
\end{abstract}

DOI: $10.1103 /$ PhysRevD.100.066001

\section{INTRODUCTION}

The Kachru-Kallosh-Linde-Trivedi (KKLT) scenario [1] provided the first construction of de Sitter (dS) vacua in string theory. The very existence of such solutions in quantum gravity has recently been questioned; see Refs. [2-4] for review articles and references. However, there is so far no generally agreed-upon flaw in the KKLT scenario and some past criticisms have already been refuted. Given this status, it is important to improve our understanding of the KKLT setup further. One such line of research has focused on the description of the anti-D3brane that provides the uplift from a supersymmetric antide Sitter (AdS) vacuum to a dS vacuum. In particular, it has become apparent in the last few years that one can describe the anti-D3-brane in terms of a four-dimensional (4D) $\mathcal{N}=1$ supergravity (SUGRA) action. In this paper, we continue this endeavor by deriving the complete $4 \mathrm{D} \mathcal{N}=1$ supergravity effective action for the KKLT scenario, including the anti-D3-brane and all of its world volume fields.

\footnotetext{
*niccolo.cribiori@tuwien.ac.at

†christoph.roupec@tuwien.ac.at

timm.wrase@tuwien.ac.at

§yamada@resceu.s.u-tokyo.ac.jp
}

Published by the American Physical Society under the terms of the Creative Commons Attribution 4.0 International license. Further distribution of this work must maintain attribution to the author(s) and the published article's title, journal citation, and DOI. Funded by SCOAP.
Our supersymmetric low-energy effective action shows that supersymmetry in the KKLT setup is spontaneously broken. While this might have been expected because the anti-D3-branes used as the uplift in the KKLT scenario are an excited state in a supersymmetric theory [5], it was not until 2014 that it was understood how to write down a supergravity action that reproduces the anti-D3-brane uplift term [6]. The connection of this uplift term to the anti-D3brane in the KKLT setup was then clarified in Refs. [7-10].

The subject of brane supersymmetry breaking started with Refs. [11-15], and the connection to nonlinear supersymmetry was first studied in Refs. [16,17]. All of these developments have broadened into a variety of different research directions and led to many interesting related results during the last few years; see, e.g., Refs. [1832]. However, so far nobody has succeeded in writing down the full four-dimensional low-energy effective supergravity action that includes all anti-D3-brane world-volume fields in the KKLT background. This action consists of a bosonic part-containing the three complex world-volume scalars and the U(1) gauge field - and a fermionic part containing the four 4D fermions. For flux compactifications, this fermionic world-volume action is currently only known to quadratic order in the fermions [33-38].

While the bosonic action seems at first to be the easier part, it is actually the fermionic action that has been studied most often over the last few years [7-10,20,27]. In particular, it has been shown that one can do an orientifold projection that removes all of the bosonic degrees of freedom (d.o.f.) from the anti-D3-brane. The fermions 
together with the bosonic uplift term can then be combined into a Volkov-Akulov type action [39] and be described in the $4 \mathrm{D} \mathcal{N}=1$ supergravity action via constrained chiral multiplets. Recently, the complete action for the GiddingsKachru-Polchinski (GKP) background fields and the four anti-D3-brane world-volume fermions has been derived in [27]. Here we extend this work by studying the full KKLT background and by including also the world-volume scalar fields and the U(1) gauge vector. Thus, we derive the complete low-energy effective supergravity action for an anti-D3-brane in the KKLT background.

The organization of the paper is as follows. In Sec. II we review the action for an anti-D3-brane in the GKP and KKLT backgrounds. In Sec. III we discuss the constrained multiplets in $4 \mathrm{D} \mathcal{N}=1$ supergravity that we need to describe the anti-D3-brane. In Sec. IV we derive the four-dimensional $\mathcal{N}=1$ supergravity action for an antiD3-brane in the KKLT background. We summarize our findings in Sec. V and we draw the conclusions in Sec. VI. Two Appendixes provide technical details.

\section{THE ANTI-D3-BRANE ACTION IN THE GKP AND KKLT BACKGROUND}

In this section we will review and (re)derive the action for an anti-D3-brane in the GKP [40] and KKLT [1] backgrounds. While many aspects of this action have been studied before, we will include here all world-volume fields of the anti-D3-brane and their couplings to the background moduli fields, which are the axiodilaton $\tau=C_{0}+\mathrm{i} e^{-\phi}$, the single Kähler modulus $T$, and the complex structure moduli $U^{A}$. The D $p$-brane action in flux backgrounds is only understood up to quadratic order in the fermions [33-38]. The known pieces of the action therefore include a bosonic part and a fermionic part that is quadratic in the worldvolume fermions. ${ }^{1}$ We will discuss these separately in the following two subsections.

\section{A. The bosonic action}

The bosonic anti-D3-brane action is the sum of the Dirac-Born-Infeld (DBI) action and the Chern-Simons (CS) action and is given in the 4D Einstein frame by

$$
\begin{gathered}
S_{\mathrm{bos}}^{\overline{D 3}}=S^{\mathrm{DBI}}+S^{\mathrm{CS}}, \\
S^{\mathrm{DBI}}=-\int d^{4} x \sqrt{-\operatorname{det}\left(\mathrm{P}\left[g_{\mu \nu}+e^{-\frac{\phi}{2}} B_{\mu \nu}\right]+e^{-\frac{\phi}{2}} F_{\mu \nu}\right)}, \\
S^{\mathrm{CS}}=-\int \mathrm{P}\left[\left(C_{0}+C_{2}+C_{4}\right) \wedge e^{B_{2}}\right] \wedge e^{F} .
\end{gathered}
$$

Here we have set $l_{s}=2 \pi \sqrt{\alpha^{\prime}}=1$, while $B_{2}$ denotes the Neveu-Schwarz Neveu-Schwarz (NSNS) KalbRamond field, $F_{\mu \nu}$ is the field strength of the U(1) gauge field living on the brane, and $\mathrm{P}$ is the pullback to the brane world volume. To simplify the presentation, we have rescaled the $U(1)$ field strength by $2 \pi$ with respect to the textbook by Polchinski [41], i.e., $F_{\mu \nu}^{\text {Polchinski }}=2 \pi F_{\mu \nu}^{\mathrm{us}}$. We have also rescaled the action by $1 / 2 \pi$ to remove the brane tension $T_{3}=(2 \pi)^{-3}\left(\alpha^{\prime}\right)^{-2}=2 \pi$.

In a GKP background [40] the metric is warped and the presence of warping makes the identification of the Kähler modulus, $\operatorname{Im}(T)$ in our case, rather cumbersome [42]. For a single Kähler modulus there is a fixed overall scaling with respect to the volume for all of the terms in the action. We can identify this scaling by working with the following metric in the Einstein frame $[43,44]$ :

$$
d s^{2}=e^{-6 u(x)}\left(1+\frac{e^{-4 \mathcal{A}(z)}}{e^{4 u(x)}}\right)^{-\frac{1}{2}} g_{\mu \nu} d x^{\mu} d x^{\nu}+e^{2 u(x)}\left(1+\frac{e^{-4 \mathcal{A}(z)}}{e^{4 u(x)}}\right)^{\frac{1}{6}} g_{a \bar{b}} d z^{a} d z^{\bar{b}},
$$

where the external indices are labeled by $\mu, \nu=0,1,2,3$, the internal indices are labeled by $a, \bar{b}=1,2,3$, and $e^{6 u}=\operatorname{vol}_{6}$ is the volume of the internal manifold, whose dependence on $\operatorname{Im}(T)$ is going to be specified below. ${ }^{2}$ This metric interpolates between the unwarped bulk region and the warped throat. We will be interested in the strong-warping regime, namely, $e^{-4 \mathcal{A}} \gg e^{4 u}$, where the metric reduces to

$$
d s^{2}=e^{2 \mathcal{A}(z)-4 u(x)} g_{\mu \nu} d x^{\mu} d x^{\nu}+e^{\frac{4}{3} u(x)-\frac{2}{3} \mathcal{A}(z)} g_{a \bar{b}} d z^{a} d z^{\bar{b}}
$$

\footnotetext{
${ }^{1}$ This action does not include terms that are linear in a world-volume fermion and a closed-string fermion.

${ }^{2}$ The ansatz (2.4) does not solve the mixed components of the ten-dimensional Einstein equations with one internal and one external index and one has to introduce a compensator field [42]. This subtlety will not affect our result. However, it would be important to confirm this explicitly by doing a proper dimensional reduction of the anti-D3-brane, extending the result for a supersymmetric D3-brane of Ref. [45].
} 
We can now proceed and start to evaluate the DBI action. Following Ref. [46], this becomes ${ }^{3}$

$$
S^{\mathrm{DBI}}=-\int d^{4} x \sqrt{-g_{4}}\left(e^{4 \mathcal{A}(H, \bar{H})-8 u(x)}+\frac{1}{2} e^{\frac{4}{3} \mathcal{A}(H, \bar{H})-\frac{8}{3} u(x)} g_{a \bar{b}}(H, \bar{H}) \partial_{\mu} H^{a} \partial^{\mu} \bar{H}^{\bar{b}}+\frac{e^{-\phi(H, \bar{H})}}{4} F_{\mu \nu} F^{\mu \nu}+\ldots\right),
$$

where the dots denote higher-order terms. These are corrections, which are small with respect to the couplings that we wrote down explicitly. The warp factor, the internal metric, and the dilaton are functions of the world-volume scalars $H^{a}$ that indicate the position of the brane and that enter the action via the pullback P. We will assume that the brane sits at some position in the strongly warped region, but we will not need to specify it further. For the rest of our discussion we will consider small fluctuations around such a position and we indicate them with the same symbol $H^{a}$ for convenience.

The kinetic term for the scalar fields arises entirely from the DBI part of the action and is therefore the same for D3branes and anti-D3-branes. The rewriting of this term in 4D $\mathcal{N}=1$ supergravity was first discussed in Ref. [47]. There it was argued that such a kinetic term stems from a Kähler potential of the type

$$
K=-3 \log [-\mathrm{i}(T-\bar{T})+k(H, \bar{H})],
$$

where $T$ is our single Kähler modulus and $k(H, \bar{H})$ is the Kähler potential corresponding to the internal Calabi-Yau metric $\partial_{H^{a}} \partial_{\bar{H}^{\bar{b}}} k(H, \bar{H}) \approx \frac{1}{6} e^{\frac{4}{3}(\mathcal{A}+u)} g_{a \bar{b}}$, where we neglected subleading terms (cf. Appendix B of Ref. [48]). The Kähler potential $k(H, \bar{H})$ does not break the no-scale structure and enters the expression of the overall volume, which indeed depends on the open- and closed-string moduli via

$$
\operatorname{vol}_{6}=e^{6 u}=(-\mathrm{i}(T-\bar{T})+k(H, \bar{H}))^{\frac{3}{2}} .
$$

The DBI action gives also rise to a scalar potential and a standard Maxwell term for the U(1) gauge field, with a coupling constant determined by $\operatorname{Im}(\tau)=e^{-\phi}$ evaluated at the position of the brane. We will discuss both of these terms further when we combine them with the CS action.

We now look at the CS action for the anti-D3-brane. In the GKP background it reduces to

$$
\begin{aligned}
S^{\mathrm{CS}} & =-\int\left(\frac{1}{2} C_{0}(H, \bar{H}) F \wedge F+C_{4}(H, \bar{H})\right) \\
& =-\int\left(\frac{1}{2} C_{0}(0,0) F \wedge F+C_{4}(0,0)+\ldots\right) \\
& =-\int d^{4} x \sqrt{-g_{4}}\left(-\frac{\operatorname{Re}(\tau)}{8} \frac{\epsilon^{\mu \nu \rho \sigma}}{\sqrt{-g_{4}}} F_{\mu \nu} F_{\rho \sigma}+\alpha(H, \bar{H})+\ldots\right),
\end{aligned}
$$

\footnotetext{
${ }^{3}$ This action can be recast in the conventions used in Ref. [27] by sending $\mathcal{A} \rightarrow \mathcal{A}-u$ and then $g_{a \bar{b}} \rightarrow g_{a \bar{b}} e^{-\frac{4}{3} \mathcal{A}}$.
}

where in the second line we expanded around the position of the brane, $H^{a}=0$, and omitted higher-order terms. We are using the fact that $\operatorname{Re}(\tau)=C_{0}(0,0)$ and $C_{4}=$ $\alpha(z, \bar{z}) \sqrt{-g_{4}} d x^{0} \wedge d x^{1} \wedge d x^{2} \wedge d x^{3}$, where $g_{4}$ is the determinant of the unwarped four-dimensional metric. Recall that $C_{2}$ and $B_{2}$ with indices along the noncompact spacetime directions are projected out by the orientifold projection.

A D3-brane in the background we are considering preserves linear $\mathcal{N}=1$ supersymmetry in 4D. The $\mathrm{U}(1)$ gauge field on its world volume has a gauge kinetic function given by $f(\tau)=-\mathrm{i} \tau$. This function is and has to be holomorphic and depends only on the axiodilaton modulus $\tau$. The real part, $\operatorname{Re}(f(\tau))=\operatorname{Im}(\tau)=e^{-\phi}$, controls the coupling in the Maxwell term and the imaginary part, $\operatorname{Im}(f(\tau))=-\operatorname{Re}(\tau)=-C_{0}$, controls the theta term. This leads to an immediate problem in the case of the antiD3-brane. With respect to the D3-brane, the anti-D3-brane has a sign difference in the CS action and thus, in order to get the correct sign for the theta term in Eq. (2.9), it seems that we would have to make the gauge kinetic function antiholomorphic, $f(\bar{\tau})=\mathrm{i} \bar{\tau}$. However, this would not be compatible with supersymmetry, since the closed-string field $\tau$ is part of an unconstrained chiral multiplet. We will show how to resolve this puzzle and maintain a holomorphic gauge kinetic function in Sec. IV. The crucial point will be that, in the background we are considering, an antiD3-brane preserves nonlinear $\mathcal{N}=1$ supersymmetry in 4D.

We can now put together the two pieces and obtain the bosonic action of the anti-D3-brane. The second term in the CS action (2.9) combines with the first term in the DBI action (2.6) into what is usually denoted by

$$
\Phi_{ \pm} \equiv e^{4 \mathcal{A}(H, \bar{H})-8 u} \pm \alpha(H, \bar{H})
$$

with the plus being for the anti-D3-brane and the minus for the D3-brane. The equations of motion in the GKP solution enforce $\Phi_{-}=0$, so that the potential for a D3-brane, $V_{D 3}=\Phi_{-}$, vanishes because the DBI part and the CS part exactly cancel. For an anti-D3-brane the contributions simply add up and we have 


$$
\begin{aligned}
S_{\mathrm{bos}}^{\overline{D 3}} & =S^{\mathrm{DBI}}+S^{\mathrm{CS}} \\
& =-\int d^{4} x \sqrt{-g_{4}}\left(2 e^{4 \mathcal{A}(H, \bar{H})-8 u}+\frac{1}{2} e^{\frac{4}{3} \mathcal{A}(H, \bar{H})-\frac{8}{3} u(x)} g_{a \bar{b}} \partial_{\mu} H^{a} \partial^{\mu} \bar{H}^{\bar{b}}+\frac{\operatorname{Im}(\tau)}{4} F_{\mu \nu} F^{\mu \nu}-\frac{\operatorname{Re}(\tau)}{8} \frac{\epsilon^{\mu \nu \rho \sigma}}{\sqrt{-g_{4}}} F_{\mu \nu} F_{\rho \sigma}+\ldots\right),
\end{aligned}
$$

where we can identify the scalar potential

$$
V_{\overline{D 3}}(H, \bar{H})=\Phi_{+}=2 e^{4 \mathcal{A}(H, \bar{H})-8 u} .
$$

Since the warp factor is minimized at the bottom of the warped throat, this is also the point where the anti-D3-brane potential is minimized and that the brane is dynamically attracted to. We can then expand the scalar potential around this point to obtain

$$
V_{\overline{D 3}}(H, \bar{H})=2 e^{4 \mathcal{A}_{0}-8 u_{0}}\left(1+\left.4 H^{a} \bar{H}^{\bar{b}} \partial_{H^{a}} \partial_{\bar{H}^{\bar{b}}} \mathcal{A}\right|_{H=0}+\left.2 H^{a} H^{b} \partial_{H^{a}} \partial_{H^{b}} \mathcal{A}\right|_{H=0}+\left.2 \bar{H}^{\bar{a}} \bar{H}^{\bar{b}} \partial_{\bar{H}^{\bar{a}}} \partial_{\bar{H}^{\bar{b}}} \mathcal{A}\right|_{H=0}+\ldots\right),
$$

where $\left.\mathcal{A}_{0} \equiv \mathcal{A}\right|_{H=0},\left.u_{0} \equiv u\right|_{H=0}$ and the dots stand for higher-order terms, which are actually suppressed by the string scale (following the same logic as in Sec. 3.1 of Ref. [46]). The first contribution in the expansion is the uplift term for an anti-D3-brane which, in a highly warped region, scales like $1 /\left(\operatorname{vol}_{6}\right)^{\frac{4}{3}}[49]{ }^{4}$ In Sec. IV we will show how to reproduce the above scalar potential using a modified version of the Kähler potential given in Eq. (2.7).

In the GKP solution the volume direction is a flat direction. The nonvanishing scalar potential for the antiD3-brane in Eq. (2.12), which is proportional to $1 /\left(\operatorname{vol}_{6}\right)^{\frac{4}{3}}$, would then lead to a runaway for the Kähler modulus. In order to avoid this, the KKLT scenario [1] includes a nonperturbative correction that can arise from either Euclidean D3-branes or a gaugino condensate on a stack of D7-branes. The effect of this nonperturbative contribution on the background as well as the anti-D3-brane uplift has recently received considerable attention [50-61]. At the heart of this discussion is the question of whether the gaugino condensation on a stack of D7-branes can be described in ten dimensions and, if that is the case, what the detailed backreaction of the gaugino condensate on the anti-D3-brane is. When the gaugino condensation or Euclidean D3-brane is taken into account, the KKLT background will have an extra term in the superpotential of the form

$$
W_{n p}=A e^{\mathrm{i} a T},
$$

where $A$ is a function that generically depends on the antiD3-brane fields. Since the gaugino condensation or Euclidean D3-brane arise from the Calabi-Yau bulk region, while we are studying an anti-D3-brane sitting at the bottom of a highly warped throat, these additional terms are expected to be highly suppressed compared to the

\footnotetext{
${ }^{4}$ In the unwarped region the uplift term actually scales like $1 /\left(\operatorname{vol}_{6}\right)^{2}[1]$.
}

tree-level potential in Eq. (2.12). After a lively debate in the recent literature [50-61], there seems to be some consensus that this is indeed the case. For this reason we will neglect these corrections here, which is in some sense generic. ${ }^{5}$ However, many throats (like, e.g., the Klebanov-Strassler geometry [62]) have isometries so that the scalar potential in Eq. (2.12) can have flat directions that would be lifted by higher-order corrections. Such higher-order corrections could arise in particular setups from the superpotential in Eq. (2.14). Such light moduli arising from the anti-D3brane were first studied in Ref. [63], and it would be interesting to study this in more detail for concrete setups using, e.g., the tools developed in Ref. [64].

\section{B. The fermionic action}

The fermionic part of the action plays a crucial role in understanding the low-energy effective description of the anti-D3-brane in the GKP or KKLT background. The reason for this is that the anti-D3-brane breaks supersymmetry spontaneously and one (combination) of the worldvolume fermions has to be the Goldstino. As we will explain in the next section, this Goldstino can be described in terms of a nilpotent chiral multiplet that couples to the standard four-dimensional $\mathcal{N}=1$ supergravity theory one obtains from the closed-string sector.

How the anti-D3-brane provides the Goldstino is not straightforward and requires some explanation. We will therefore comment on this before actually presenting the action. The fermionic action for a $D p$-brane in a flux background is only known to quadratic order in the fermions [33-38] and the anti-D3-brane has been studied in this context in Refs. [7,8,27,46]. In particular, the four world-volume fermions on the anti-D3-brane can be divided into $\lambda$, which is a singlet under the $\mathrm{SU}(3)$ holonomy

\footnotetext{
${ }^{5}$ For a supersymmetric D3-brane the tree-level scalar potential vanishes and these terms provide a very interesting, small potential that was first studied in Ref. [48].
} 
group of the internal manifold, and $\chi^{i}$, with $i=1,2,3$, which transform as a triplet. The masses and some of the couplings of these fermions are controlled by the imaginary self-dual (ISD) part

$$
G_{3}^{\mathrm{ISD}}=\frac{1}{2}\left(G_{3}-\mathrm{i} *_{6} G_{3}\right)
$$

of the background flux $G_{3}=F_{3}-\mathrm{i} e^{-\phi} H_{3}[8,46]$, where in our conventions $F_{3}=d C_{2}-C_{0} H_{3}$. In particular, the mass of the singlet arises from a flux of $(0,3)$ type, the interaction between $\lambda$ and the $\chi^{i}$ is proportional to a nonprimitive $(1,2)$ flux, and the masses of the $\chi^{i}$ are determined by a primitive $(2,1)$ flux. In a supersymmetric GKP background, $\lambda$ is the Goldstino and correspondingly it does not mix with the $\chi^{i}$ and has no mass term. This is consistent with the fact that the anti-D3-brane is the only source of supersymmetry breaking in this context [8].

In a nonsupersymmetric GKP background we have a nonvanishing Gukov-Vafa-Witten superpotential [65]

$$
W_{\mathrm{GVW}}=\int G_{3} \wedge \Omega \neq 0,
$$

where $\Omega$ is the (3,0)-form of the Calabi-Yau manifold, and therefore we get a nonvanishing $F$-term for the Kähler modulus $T$,

$$
D_{T} W_{\mathrm{GVW}}=K_{T} W_{\mathrm{GVW}} \neq 0 .
$$

As a consequence, in a nonsupersymmetric GKP background with Eq. (2.16), the $G_{3}$ flux must contain a nonvanishing $(0,3)$ piece and the background itself breaks supersymmetry spontaneously. In such a situation, the Goldstino is a closed-string fermion. If we add an antiD3-brane to this background, then the singlet $\lambda$ will also have a mass and cannot (and does not have to) be the Goldstino. Indeed, since both the anti-D3-brane as well as the background $(0,3) G_{3}$ flux break supersymmetry spontaneously, the Goldstino is expected to be a linear combination of $\lambda$ and the closed-string fermion which was the Goldstino before the addition of the anti-D3-brane.

The actual KKLT background of interest has, besides a nonvanishing $W_{\mathrm{GVW}}$, a nonperturbative superpotential term given in Eq. (2.14). In this case one can find a supersymmetric solution,

$$
D_{T}\left(W_{\mathrm{GVW}}+W_{n p}\right)=0,
$$

which gives $\partial_{T} W_{n p}=-K_{T}\left(W_{\mathrm{GVW}}+W_{n p}\right)$. This solution is a supersymmetric AdS vacuum that is uplifted by the anti-D3-brane to the KKLT dS vacuum. At this point, however, one might have noticed the following issue: the anti-D3-brane is actually the sole source of supersymmetry breaking and therefore it needs to provide the massless
Goldstino. On the other hand, the background has a $(2,1)$ as well as a $(0,3) G_{3}$ flux, which seem to give a mass to $\chi^{i}$ and $\lambda$ as well [8]. In other words, all fermions appear to be massive and it is not clear whether a massless Goldstino is present. The resolution of this apparent puzzle is due to the behavior of the $(0,3) G_{3}$ flux in the presence of a gaugino condensate on a stack of D7-branes. In particular, it was shown in Refs. [66,67] that the $(0,3) G_{3}$ flux localizes on top of the D7-branes that are located in the bulk of the warped Calabi-Yau manifold. Therefore, the pull-back of this $(0,3) G_{3}$ flux onto the anti-D3-brane world volume vanishes, since the anti-D3-branes sits at the bottom of a warped throat. Thus, $\lambda$ does not get a mass and is the Goldstino provided by the anti-D3-brane, which is the sole source of supersymmetry breaking. ${ }^{6}$

Having clarified how the anti-D3-brane action in the KKLT background provides the massless Goldstino plus three more massive fermions, we now work out the couplings of these fermions to the closed-string moduli $\tau, T$ and $U^{A}$. The fermionic anti-D3-brane action was studied, e.g., in Refs. $[8,27,33,46]$. The part of the action that is quadratic in world-sheet fermions is given in the Einstein frame by $[8,37,68]^{7}$

$$
\begin{aligned}
S_{\mathrm{fer}}^{\overline{D 3}}= & 2 \int d^{4} x \sqrt{-g_{4}}\left[e^{4 \mathcal{A}-8 u} \bar{\theta} \Gamma^{\mu}\left(\nabla_{\mu}-\frac{1}{4} e^{\phi} F_{\mu} \tilde{\Gamma}_{0123}\right) \theta\right. \\
& +\frac{1}{8 \cdot 4 !} \bar{\theta}\left(e^{\frac{16-A}{3}-\frac{32 u}{3}} \Gamma^{\mu m n p q} F_{\mu m n p q}\right. \\
& \left.-2 e^{\frac{8.4}{3}-\frac{16 u}{3}} \Gamma^{\mu \nu \rho m n} F_{\mu \nu \rho m n}\right) \tilde{\Gamma}_{0123} \theta \\
& \left.-\frac{\mathrm{i}}{24} e^{6 \mathcal{A}-12 u+\frac{\phi}{2}}\left(G_{m n p}^{\mathrm{ISD}}-\bar{G}_{m n p}^{\mathrm{ISD}}\right) \bar{\theta} \Gamma^{m n n} \theta\right]
\end{aligned}
$$

where $\theta$ is a 16-component Majorana-Weyl spinor of type IIB theory, the indices $m, n, \ldots=4,5, \ldots, 9$ are internal, $\Gamma_{\ldots}$ has curved but unwarped indices, and $\tilde{\Gamma}_{\ldots}$ has flat indices. Following Refs. $[8,33]$, we can decompose the spinor $\theta$ into four-dimensional Weyl spinors. In the notation of Ref. [69], we have an SU(3) singlet $P_{L} \lambda$ and a triplet $P_{L} \chi^{i}$. The reduction of the last line and the kinetic term of Eq. (2.19) was performed in detail in Ref. [8]. If the

\footnotetext{
${ }^{6}$ This is only true in the strict probe limit. Once the anti-D3brane backreacts onto the geometry via the uplift term $V_{\text {up }} \propto 1 /(-\mathrm{i}(T-\bar{T}))^{2}$, the $T$ modulus shifts away from the supersymmetric minimum. As a consequence, $D_{T} W$ will not be zero anymore and the Goldstino will be a mixture of $\lambda$ and the fermionic partner of the $T$ modulus.

${ }^{7}$ This can be obtained from the string-frame result in Refs. [8,68] with $g_{\mu \nu}^{\mathrm{S}}=e^{\frac{\phi}{2}+2 \mathcal{A}-4 u} g_{\mu \nu}$ and $\theta^{\mathrm{S}}=e^{\frac{\phi}{8}-u+\frac{\mathcal{A}}{2}} \theta$. We follow the conventions of Ref. [8], which uses the action given in Eqs. (4.4) to (4.6) of Ref. [68]. We rederived the coupling to $F_{1}$ starting from Eqs. (A.5) and (A.7) in Ref. [68] and found the opposite sign for the corresponding term. Our expression agrees with the generic four-dimensional supergravity action, as we check below.
} 
brane action is evaluated on a fixed background the remaining terms vanish. On the contrary, they should be taken into account when the background fields are dynamical, as is the case in our setup. To the best of our knowledge, these terms are worked out here for the first time.

We start by considering the contribution arising from the spin connection. In particular, we have

$$
\bar{\theta} \Gamma^{\mu} \nabla_{\mu} \theta=\bar{\theta} \Gamma^{\mu}\left(\partial_{\mu}+\frac{1}{4} \omega_{\mu}^{\tilde{a} \tilde{b}} \tilde{\Gamma}_{\tilde{a} \tilde{b}}+\frac{1}{4} \omega_{\mu}^{\bar{l}} \tilde{\Gamma}_{\overline{i l}}\right) \theta,
$$

where the flat indices take values $\tilde{a}, \tilde{b}=0,1,2,3$ and $i$, $\bar{\imath}=1,2,3$. Since the spin connection with mixed indices vanishes, $\omega_{\mu}^{\tilde{a} i}=\omega_{\mu}^{\tilde{a} \bar{\imath}}=0$, we have already omitted the corresponding terms. The first two terms in the equation above construct the $4 \mathrm{D}$ covariant derivative, while the last term gives rise to a coupling between the fermions and the complex structure moduli, which can be calculated as follows. In our setup the metric is block diagonal with a 4D part and a six-dimensional part, and therefore the vielbein is likewise block diagonal. Then, the six-dimensional internal vielbein part satisfies $e_{i}^{a} g_{a \bar{b}} e_{\bar{l}}^{\bar{b}}=\delta_{\bar{i} \bar{l}}$, so that $e_{i}^{a}$ is a function of the Kähler modulus $T$ and the complex structure moduli $U^{A}$. Since the holomorphic $(3,0)$-form $\Omega_{a b c}=\epsilon_{i j k} e_{a}^{i} e_{b}^{j} e_{c}^{k}$ only depends on the $U^{A}$ and not on the $\bar{U}^{A}$, we can conclude that $e_{a}^{i}$ and its inverse $e_{i}^{a}$ depend only on $T, \bar{T}$, and the $U^{A}$, but not on $\bar{U}^{A}$. Namely, we have

$\partial_{\mu} e_{i}^{a}=\left(\partial_{T} e_{i}^{a}\right) \partial_{\mu} T+\left(\partial_{\bar{T}} e_{i}^{a}\right) \partial_{\mu} \bar{T}+\left(\partial_{U^{A}} e_{i}^{a}\right) \partial_{\mu} U^{A}$

Having a single volume modulus, the metric and the vielbein have a simple overall volume dependence, i.e., they depend to leading order in large volume on $(T-\bar{T})$ to some power, so that $\partial_{T} e_{i}^{a}=-\partial_{\bar{T}} e_{i}^{a} \propto e_{i}^{a}$. This means that the relevant spin connection reduces to

$$
\omega_{\mu}^{i \bar{l}}=e^{\bar{a} i} \partial_{\mu} e_{\bar{a}}^{\bar{l}}-e^{a \bar{u}} \partial_{\mu} e_{a}^{i}=e^{\bar{a} i}\left(\partial_{\bar{U}^{A}} e_{\bar{a}}^{\bar{i}}\right) \partial_{\mu} \bar{U}^{A}-e^{a \bar{u}}\left(\partial_{U^{A}} e_{a}^{i}\right) \partial_{\mu} U^{A} .
$$

We will now use the fact that we have only a single Kähler modulus and correspondingly only a single $(1,1)$-form to substantially simplify the above expression. In particular, from the spin connection we can define a 2-form that has to be proportional to the Kähler form $J$, or in flat indices to $\delta_{\overline{l i}}$, if it is in cohomology ${ }^{8}$

$$
\omega_{\mu \bar{i}} e_{a}^{i} e_{\bar{b}}^{\bar{l}} \propto \mathrm{i} J_{a \bar{b}}=\delta_{\overline{i l}} e_{a}^{i} e_{\bar{b}}^{\bar{i}} .
$$

We can then write

$$
\omega_{\mu}^{i \bar{i}} \tilde{\Gamma}_{\bar{\imath}}=\frac{1}{3} \omega_{\mu}^{i \bar{i}} \delta_{i \bar{l}} \delta^{j \bar{j}} \tilde{\Gamma}_{j \bar{\jmath}}
$$

and the first new fermionic contribution to the 4D anti-D3-brane action is

$$
\begin{aligned}
& \bar{\theta} \Gamma^{\mu} \omega_{\mu}^{i \bar{l}} \tilde{\Gamma}_{\overline{i l}} \theta=\frac{1}{3} \bar{\theta} \Gamma^{\mu} \omega_{\mu}^{i \bar{l}} \delta_{i \bar{l}} \delta^{j \bar{\jmath}} \tilde{\Gamma}_{j \bar{j}} \theta=\frac{1}{3} \omega_{\mu}^{k \bar{k}} \delta_{k \bar{k}}\left(3 \bar{\lambda} P_{R} \gamma^{\mu} \lambda-\delta_{i \bar{\jmath}} \bar{\chi}^{\bar{\jmath}} P_{R} \gamma^{\mu} \chi^{i}\right) \\
& =\frac{1}{3} \delta_{\bar{i}}\left(e^{\bar{a} i}\left(\partial_{\bar{U}^{A}} e_{\bar{a}}^{\bar{\imath}}\right) \partial_{\mu} \bar{U}^{A}-e^{a \bar{\imath}}\left(\partial_{U^{A}} e_{a}^{i}\right) \partial_{\mu} U^{A}\right)\left(3 \bar{\lambda} P_{R} \gamma^{\mu} \lambda-\delta_{j \bar{\jmath}} \bar{\chi}^{\bar{j}} P_{R} \gamma^{\mu} \chi^{j}\right) .
\end{aligned}
$$

The second new term involves a coupling to the derivative $F_{\mu}=\partial_{\mu} C_{0}=\partial_{\mu} \operatorname{Re} \tau$. Its calculation is simpler with respect to the previous case and it gives directly

$$
e^{\phi} F_{\mu} \bar{\theta} \Gamma^{\mu} \tilde{\Gamma}_{0123} \theta=\frac{\partial_{\mu} \operatorname{Re}(\tau)}{\operatorname{Im}(\tau)} \bar{\theta} \Gamma^{\mu} \tilde{\Gamma}_{0123} \theta=-\mathrm{i} \frac{\partial_{\mu} \operatorname{Re}(\tau)}{\operatorname{Im}(\tau)}\left(\bar{\lambda} P_{R} \gamma^{\mu} \lambda+\delta_{i \bar{j}} \bar{\chi}^{\bar{\jmath}} P_{R} \gamma^{\mu} \chi^{i}\right)
$$

The last contribution in Eq. (2.19) that we have to calculate is a derivative coupling to the $C_{4}$ axion. To this purpose, we recall that for a Calabi-Yau manifold with a single Kähler modulus $T$, there is only a single (2,2)-form that we denote by $Y_{2,2}$ and that is normalized such that it integrates to one on the single 4-cycle $\Sigma_{4}$. In particular, the Kähler modulus is constructed out of the 4-forms $C_{4}$ and $J \wedge J$ and it has to be holomorphic, since it is described by a chiral multiplet in four dimensions. We can therefore decompose it on the basis given by $Y_{2,2}$ and find

\footnotetext{
${ }^{8}$ It is not clear to us that this 2-form indeed has to be in cohomology for generic $\mathrm{CY}_{3}$ manifolds. For toroidal orbifold examples this is indeed the case, but we lack a generic argument. The following is therefore not a strict mathematical proof. We thank Harald Skarke for discussing this point.
} 


$$
T \equiv \int_{\Sigma_{4}}\left(C_{4}-\frac{\mathrm{i}}{2} J \wedge J\right)=\int_{\Sigma_{4}} c_{4}\left(x^{\mu}\right) Y_{2,2}+\mathrm{i} \operatorname{Im}(T) \int_{\Sigma_{4}} Y_{2,2}
$$

From the above we can directly identify $C_{4} \supset c_{4}\left(x^{\mu}\right) Y_{2,2}=-c_{4}\left(x^{\mu}\right) \frac{J \wedge J}{2 \operatorname{Im}(T)}$. Using the fact that $i e_{i}^{u} e_{\bar{l}}^{\bar{u}} J_{u \bar{u}}=\delta_{\bar{l}}$, where $u, \bar{u}$ are curved and warped indices, while recalling that the matrix $\Gamma^{\mu n p q r}$ appearing in Eq. (2.19) has real curved but unwarped indices, we can finally calculate the desired term,

$$
\begin{aligned}
\frac{1}{4 !} e^{\frac{4 A}{3}-\frac{8 u}{3}} \bar{\theta} \Gamma^{\mu n p q r} \tilde{\Gamma}_{0123} F_{\mu n p q r} \theta & =\frac{\partial_{\mu} \operatorname{Re}(T)}{2 \operatorname{Im}(T)} \bar{\theta} \delta_{i \bar{l}} \delta_{j \bar{j}} \Gamma^{\mu} \tilde{\Gamma}^{i \bar{i} j} \tilde{\Gamma}_{0123} \theta \\
& =-\mathrm{i} \frac{\partial_{\mu} \operatorname{Re}(T)}{\operatorname{Im}(T)}\left(3 \bar{\lambda} P_{R} \gamma^{\mu} \lambda-\delta_{i \bar{j}} \bar{\chi}^{\bar{\jmath}} P_{R} \gamma^{\mu} \chi^{i}\right)
\end{aligned}
$$

Actually, this is only one of the two contributions containing $C_{4}$ and appearing in Eq. (2.19). However, due to the self-duality of $d C_{4}$ in ten dimensions, one finds that the four-dimensional 2-form $C_{4} \supset c_{4, \mu \nu}^{(2)} d x^{\mu} \wedge d x^{\nu} \wedge$ $Y_{1,1}$ is dual in four dimensions to $c_{4}\left(x^{\mu}\right)$, so that the two corresponding terms in the 3-brane action are equal, namely,

$$
F_{\mu n p q r} \Gamma^{\mu n p q r}=-2 e^{-\frac{8 A}{3}+\frac{16 u}{3}} F_{\mu \nu \rho m n} \Gamma^{\mu \nu \rho m n} \tilde{\Gamma}_{*},
$$

and therefore the two terms in Eq. (2.19) add up.

Up to total derivatives and written in terms of four-dimensional spinors, the action (2.19) then becomes

$$
\begin{aligned}
S_{\mathrm{fer}}^{\overline{D 3}}= & 2 \int d^{4} x \sqrt{-g_{4}} e^{4 \mathcal{A}-8 u}\left[\bar{\lambda} P_{R} \gamma^{\mu} \nabla_{\mu} \lambda+\delta_{i \bar{\jmath}} \overline{\chi^{\jmath}} P_{R} \gamma^{\mu} \nabla_{\mu} \chi^{i}+\frac{\mathrm{i}}{4 \operatorname{Im}(\tau)} \partial_{\mu} \operatorname{Re}(\tau)\left(\bar{\lambda} P_{R} \gamma^{\mu} \lambda+\delta_{i \bar{\jmath}} \bar{\chi}^{\bar{\jmath}} P_{R} \gamma^{\mu} \chi^{i}\right)\right. \\
& -\frac{\mathrm{i}}{4 \operatorname{Im}(T)} \partial_{\mu} \operatorname{Re}(T)\left(3 \bar{\lambda} P_{R} \gamma^{\mu} \lambda-\delta_{i \bar{\jmath}} \bar{\chi}^{\bar{\jmath}} P_{R} \gamma^{\mu} \chi^{i}\right)+\frac{1}{12} \omega_{\mu}^{k \bar{k}} \delta_{k \bar{k}}\left(3 \bar{\lambda} P_{R} \gamma^{\mu} \lambda-\delta_{i \bar{\jmath}} \bar{\chi}^{\bar{\jmath}} P_{R} \gamma^{\mu} \chi^{i}\right) \\
& \left.+\left(\frac{1}{2} m \bar{\lambda} P_{L} \lambda+m_{i} \bar{\lambda} P_{L} \chi^{i}+\frac{1}{2} m_{i j} \bar{\chi}^{i} P_{L} \chi^{j}+\text { c.c. }\right)\right]
\end{aligned}
$$

The masses depend on the $G_{3}^{\mathrm{ISD}}$ flux as

$$
\begin{gathered}
m=\frac{\sqrt{2}}{12} \mathrm{i} e^{2 \mathcal{A}-4 u+\frac{\phi}{2}} \bar{\Omega}^{a b c} \bar{G}_{a b c}^{\mathrm{ISD}}, \\
m_{i}=-\frac{\sqrt{2}}{4} e^{2 \mathcal{A}-4 u+\frac{\phi}{2}} e_{i}^{a} \bar{G}_{a b \bar{c}}^{\mathrm{ISD}} J^{b \bar{c}} \\
m_{i j}=\frac{\sqrt{2}}{8} \mathrm{i} e^{2 \mathcal{A}-4 u+\frac{\phi}{2}}\left(e_{i}^{c} e_{j}^{d}+e_{j}^{c} e_{i}^{d}\right) \Omega_{a b c} g^{a \bar{a}} g^{b \bar{b}} \bar{G}_{d \bar{a} \bar{b}}^{\mathrm{ISD}} .
\end{gathered}
$$

As we discussed at the beginning of this subsection, in a GKP background or in the KKLT setup with gaugino condensation on a stack of D7-branes in the bulk that is away from the anti-D3-brane, the pullback of the $(0,3)$ part of the $G_{3}$ flux onto the anti-D3-brane world volume vanishes. In addition, in the same background the $(2,1)$ part of $G_{3}^{\mathrm{ISD}}$ is primitive, namely, $G_{a b \bar{c}}^{\mathrm{ISD}} J^{b \bar{c}}=0$, where $J_{a \bar{b}}$ is the Kähler form on the Calabi-Yau. As a consequence of these two facts we have that

$$
m=0=m_{i}
$$

and then the singlet fermion $\lambda$ remains massless, while the $\chi^{i}$ generically have nonvanishing mass terms. $\lambda$ will therefore be the Goldstino associated to the broken supersymmetry. It arises from the brane, as is expected for an anti-D3-brane added to a supersymmetric background. 
Finally, the complete anti-D3-brane action in the KKLT background is

$$
\begin{aligned}
S^{\overline{D 3}}= & S_{\mathrm{bos}}^{\overline{D 3}}+S_{\mathrm{fer}}^{\overline{D 3}} \\
= & -\int d^{4} x \sqrt{-g_{4}}\left(2 e^{4 \mathcal{A}-8 u}+\frac{1}{2} e^{\frac{4}{3} \mathcal{A}-\frac{8}{3} u} g_{a \bar{b}} \partial_{\mu} H^{a} \partial^{\mu} \bar{H}^{\bar{b}}+\frac{\operatorname{Im}(\tau)}{4} F_{\mu \nu} F^{\mu \nu}-\frac{\operatorname{Re}(\tau)}{8} \frac{\epsilon^{\mu \nu \rho \sigma}}{\sqrt{-g_{4}}} F_{\mu \nu} F_{\rho \sigma}\right) \\
& +2 \int d^{4} x \sqrt{-g_{4}} e^{4 \mathcal{A}-8 u}\left[\bar{\lambda} P_{R} \gamma^{\mu} \nabla_{\mu} \lambda+\delta_{i \bar{\jmath}} \bar{\chi}^{\bar{\jmath}} P_{R} \gamma^{\mu} \nabla_{\mu} \chi^{i}+\frac{\mathrm{i}}{4 \operatorname{Im}(\tau)} \partial_{\mu} \operatorname{Re}(\tau)\left(\bar{\lambda} P_{R} \gamma^{\mu} \lambda+\delta_{i \bar{j}} \bar{\chi}^{\bar{\jmath}} P_{R} \gamma^{\mu} \chi^{i}\right)\right. \\
& -\frac{\mathrm{i}}{4 \operatorname{Im}(T)} \partial_{\mu} \operatorname{Re}(T)\left(3 \bar{\lambda} P_{R} \gamma^{\mu} \lambda-\delta_{i \bar{\jmath}} \bar{\chi}^{\bar{j}} P_{R} \gamma^{\mu} \chi^{i}\right)+\frac{1}{12} \omega_{\mu}^{k \bar{k}} \delta_{k \bar{k}}\left(3 \bar{\lambda} P_{R} \gamma^{\mu} \lambda-\delta_{i \bar{\jmath}} \bar{\chi}^{\bar{\jmath}} P_{R} \gamma^{\mu} \chi^{i}\right) \\
& \left.+\frac{1}{2} m_{i j} \bar{\chi}^{i} P_{L} \chi^{j}+\frac{1}{2} \bar{m}_{\bar{\imath} \bar{\jmath}} \bar{\chi}^{\bar{\imath}} P_{R} \chi^{\bar{\jmath}}\right] .
\end{aligned}
$$

\section{The supersymmetric D3-brane action}

The above action is the leading-order component action for an anti-D3-brane in the KKLT or GKP background and we will show how to rewrite it in terms of $\mathcal{N}=1$ supergravity in Sec. IV. However, before doing so, it is instructive to perform a simple check on our result and compare it to the known couplings in the D3-brane action. We devote the present subsection to this purpose.
Recall that the D3-brane differs from the anti-D3-brane action by a sign flip of the Ramond-Ramond (RR) fields. In the supersymmetric D3-brane case one also has to take into account that the first "uplift term" in the bosonic action and the fermionic mass terms vanish. What remains is then a standard $\mathcal{N}=1$ supergravity action for a single vector multiplet, containing $\lambda$ and $A_{\mu}$, and three chiral multiplets, containing $H^{a}$ and $\chi^{a} \equiv e_{i}^{a} \chi^{i}$. Explicitly, it is given by

$$
\begin{aligned}
S^{D 3}= & S_{\mathrm{bos}}^{D 3}+S_{\mathrm{fer}}^{D 3} \\
= & -\int d^{4} x \sqrt{-g_{4}}\left(\frac{1}{2} e^{\frac{4}{3} \mathcal{A}-\frac{8}{3} u} g_{a \bar{b}} \partial_{\mu} H^{a} \partial^{\mu} \bar{H}^{\bar{b}}+\frac{\operatorname{Im}(\tau)}{4} F_{\mu \nu} F^{\mu \nu}+\frac{\operatorname{Re}(\tau)}{8} \frac{\epsilon^{\mu \nu \rho \sigma}}{\sqrt{-g_{4}}} F_{\mu \nu} F_{\rho \sigma}\right) \\
& +2 \int d^{4} x \sqrt{-g_{4}} e^{4 \mathcal{A}-8 u}\left[\bar{\lambda} P_{R} \gamma^{\mu} \nabla_{\mu} \lambda+\delta_{i \bar{\jmath}} \bar{\chi}^{\bar{\jmath}} P_{R} \gamma^{\mu} \nabla_{\mu} \chi^{i}-\frac{\mathrm{i}}{4 \operatorname{Im}(\tau)} \partial_{\mu} \operatorname{Re}(\tau)\left(\bar{\lambda} P_{R} \gamma^{\mu} \lambda+\delta_{i \bar{\jmath}} \bar{\chi}^{\bar{j}} P_{R} \gamma^{\mu} \chi^{i}\right)\right. \\
& \left.+\frac{\mathrm{i}}{4 \operatorname{Im}(T)} \partial_{\mu} \operatorname{Re}(T)\left(3 \bar{\lambda} P_{R} \gamma^{\mu} \lambda-\delta_{i \bar{\jmath}} \bar{\jmath}^{\bar{\jmath}} P_{R} \gamma^{\mu} \chi^{i}\right)+\frac{1}{12} \omega_{\mu}^{k \bar{k}} \delta_{k \bar{k}}\left(3 \bar{\lambda} P_{R} \gamma^{\mu} \lambda-\delta_{i \bar{\jmath}} \bar{\chi}^{\bar{\jmath}} P_{R} \gamma^{\mu} \chi^{i}\right)\right] .
\end{aligned}
$$

It is interesting to study the derivative couplings involving (derivatives of) the closed-string axions and the open-string fermions on the supersymmetric D3-brane, since this will provide useful information about the form of the Kähler potential even for the anti-D3-brane. Let us begin with the coupling to $\partial_{\mu} \tau$. The fermions $\chi^{i}$ come in chiral multiplets and carry no U(1) charge, but via their Kähler covariant derivative they couple to all scalars. In particular, they couple to the axiodilaton $\tau$ via interactions of the type (see, e.g., chapter 18 in Ref. [69])

$$
\begin{aligned}
\mathcal{L}^{\text {SUGRA }} \supset & -\delta_{i \bar{\jmath}} \bar{\chi}^{\bar{j}} P_{R} \gamma^{\mu}\left(\partial_{\mu}-\frac{1}{4}\left[\partial_{\mu} \tau \partial_{\tau} K-\partial_{\mu} \bar{\tau} \partial_{\bar{\tau}} K\right]\right) \chi^{i} \\
& -\frac{1}{2} \delta_{i \bar{\jmath}} \bar{\chi}^{\bar{\jmath}} P_{R} \gamma^{\mu} \Gamma_{k \tau}^{i} \partial_{\mu} \tau \chi^{k}-\frac{1}{2} \delta_{i \bar{\jmath}} \bar{\chi}^{i} P_{L} \gamma^{\mu} \Gamma_{\bar{k} \bar{\tau}}^{\bar{j}} \partial_{\mu} \bar{\tau} \chi^{\bar{k}}
\end{aligned}
$$

We find that the Kähler potential $K^{(\tau)}=-\log [-\mathrm{i}(\tau-\bar{\tau})]$ leads precisely to the coupling involving $\partial_{\mu} \operatorname{Re}(\tau) / \operatorname{Im}(\tau)$ that is given in Eq. (2.36), via the two terms in square brackets above. In particular, the prefactor of this term relative to the kinetic term also agrees with the generic supergravity result. This means that the mixed Christoffel symbols $\Gamma_{j \tau}^{i}$ have to vanish, which is indeed the case for appropriately chosen Kähler potentials, like the one given in Eq. (2.7) that couples the scalars in the chiral multiplets only to $T$ and not to $\tau$.

The couplings of $\tau$ and $\lambda$ can be likewise read off from the general supergravity action. Recall that the gauge kinetic function for a D3-brane is $f(\tau)=-\mathrm{i} \tau$. The kinetic term for the gaugino is normalized so that its prefactor is $\operatorname{Re}(f)=\operatorname{Im}(\tau)=e^{-\phi}$, so we have to rescale $\lambda=e^{-\frac{\phi}{2}} \lambda^{\prime}$, but this does not lead to new derivative terms since $\bar{\lambda} \gamma^{\mu} \lambda=0$ for Majorana spinors in four dimensions. The standard supergravity action for $\lambda^{\prime}$ then contains terms of the form 


$$
\mathcal{L}^{\mathrm{SUGRA}} \supset-\frac{1}{2} \operatorname{Re}(f) \bar{\lambda}^{\prime} \gamma^{\mu}\left(\partial_{\mu}+\frac{1}{4}\left(\partial_{\mu} \tau \partial_{\tau} K-\partial_{\mu} \bar{\tau} \partial_{\bar{\tau}} K\right) \gamma_{*}\right) \lambda^{\prime}+\frac{\mathrm{i}}{4} \partial_{\mu} \operatorname{Im}(f) \bar{\lambda}^{\prime} \gamma_{*} \gamma^{\mu} \lambda^{\prime}
$$

These combine correctly to give the coupling of $\lambda$ to $\partial_{\mu} \operatorname{Re}(\tau) / \operatorname{Im}(\tau)$ that is given in Eq. (2.36).

We now proceed and look at the coupling to $\partial_{\mu} \operatorname{Re}(T)$ by performing a similar analysis. For the $\chi^{i}$ that are in chiral multiplets, these couplings arise again from the standard supergravity terms,

$$
\begin{aligned}
\mathcal{L}^{\text {SUGRA }} \supset & -\delta_{i \bar{\jmath}} \bar{\chi}^{\bar{\jmath}} P_{R} \gamma^{\mu}\left(\partial_{\mu}-\frac{1}{4}\left[\partial_{\mu} T \partial_{T} K-\partial_{\mu} \bar{T} \partial_{\bar{T}} K\right]\right) \chi^{i} \\
& -\frac{1}{2} \delta_{i \bar{\jmath}} \bar{\chi}^{\bar{\jmath}} P_{R} \gamma^{\mu} \Gamma_{k T}^{i} \partial_{\mu} T \chi^{k}-\frac{1}{2} \delta_{i \bar{\jmath}} \bar{\chi}^{i} P_{L} \gamma^{\mu} \Gamma_{\bar{k} \bar{T}}^{\bar{\jmath}} \partial_{\mu} \bar{T} \chi^{\bar{k}}
\end{aligned}
$$

This time, however, the Christoffel symbols are not vanishing. Indeed, we find for the Kähler potential in Eq. (2.7) that $\Gamma_{j T}^{i} \approx \frac{\mathrm{i}}{2 \operatorname{Im}(T)}$ in the large-volume limit, where we neglect terms involving $k(H, \bar{H})$ compared to $\operatorname{Im}(T)$. These Christoffel symbols are then combined with the terms involving partial derivatives of the Kähler potential $K$ to again give the terms in the component action (2.36) with the correct coefficient.

Since for the gauginos $\lambda$ there is no contribution involving the Christoffel symbols, the standard supergravity action has terms, written using $\lambda^{\prime}=e^{\frac{\phi}{2}} \lambda$, that are simply

$$
\mathcal{L}^{\mathrm{SUGRA}} \supset-\frac{1}{2} \operatorname{Re}(f) \bar{\lambda}^{\prime} \gamma^{\mu}\left(\partial_{\mu}+\frac{1}{4}\left(\partial_{\mu} T \partial_{T} K-\partial_{\mu} \bar{T} \partial_{\bar{T}} K\right) \gamma_{*}\right) \lambda^{\prime} .
$$

Again, they match the component action (2.36) and the very absence of the Christoffel terms for $\lambda$ compared to $\chi^{i}$ explains the different prefactor for the corresponding terms.

Last and most interestingly, we look at the terms involving the complex structure moduli $U^{A}$. The two terms in the spin connection in Eq. (2.22) are independent, since one is proportional to $\partial_{\mu} U^{A}$ and the other to $\partial_{\mu} \bar{U}^{A}$. Following a reasoning similar to that of the previous subsection, this means that these terms have to be both proportional to $\delta_{\bar{i}}$. Therefore, using the fact that $\partial_{\mu}\left(e_{u}^{i} e^{u \bar{\imath}}\right)=0$, we can rewrite

$$
\partial_{U^{A}} e_{u}^{i}=-e_{v}^{i}\left(\partial_{U^{A}} e^{v \bar{\imath}}\right) e_{u \bar{l}}=-\frac{1}{3} e_{v}^{j}\left(\partial_{U^{A}} e^{v \bar{\jmath}}\right) \delta_{j \bar{\jmath}} \delta^{\bar{\imath}} e_{u \bar{l}}=-\frac{1}{3} e_{v}^{j}\left(\partial_{U^{A}} e^{v \bar{\jmath}}\right) \delta_{j \bar{\jmath}} e_{u}^{i},
$$

where $u, v, \ldots=1,2,3$ are curved warped indices. This expression can be rephrased in terms of the holomorphic $(3,0)$ form $\Omega$ on the Calabi-Yau manifold by using the fact that

$$
\begin{aligned}
\partial_{U^{A}} \Omega & =\frac{1}{3 ! \cdot 3 !} \partial_{U^{A}}\left(e_{u}^{i} e_{v}^{j} e_{w}^{k} \epsilon_{i j k} d z^{u} \wedge d z^{v} \wedge d z^{w}\right) \\
& =\frac{3}{3 ! \cdot 3 !}\left(\partial_{U^{A}} e_{u}^{i}\right) e_{v}^{j} e_{w}^{k} \epsilon_{i j k} d z^{u} \wedge d z^{v} \wedge d z^{w} \\
& =-\frac{1}{3 ! \cdot 3 !} e_{t}^{l}\left(\partial_{U^{A}} e^{t \bar{\jmath}}\right) \delta_{l j} e_{u}^{i} e_{v}^{j} e_{w}^{k} \epsilon_{i j k} d z^{u} \wedge d z^{v} \wedge d z^{w} \\
& =-e_{v}^{j}\left(\partial_{U^{A}} e^{v \bar{J}}\right) \delta_{j \bar{J}} \Omega .
\end{aligned}
$$

On the other hand, we can expand $\Omega$ on a cohomology basis, namely, $\Omega=Z^{K} \alpha_{K}-F_{K} \beta^{K}$, where $Z_{K}$ and $F^{K}$ are functions of the $U^{A}$, while the $\alpha_{K}$ and $\beta^{K}$ are a basis for the 3 -forms with the only nonvanishing integrals $\int \alpha_{K} \wedge \beta^{L}=\delta_{K}^{L}$. In particular, it follows from Eq. (2.42) that

$$
\partial_{U^{A}} Z^{K}=-e_{v}^{j}\left(\partial_{U^{A}} e^{v \bar{J}}\right) \delta_{j \bar{j}} Z^{K}, \quad \partial_{U^{A}} F_{K}=-e_{v}^{j}\left(\partial_{U^{A}} e^{v \bar{J}}\right) \delta_{j \bar{j}} F_{K} .
$$

Now we introduce the Kähler potential for the complex structure moduli,

$$
K^{(U)}=-\log \left[-\mathrm{i} \int \Omega \wedge \bar{\Omega}\right]=-\log \left[\mathrm{i}\left(Z^{K} \bar{F}_{K}-\bar{Z}^{K} F_{K}\right)\right],
$$


and we can use this to eventually find

$$
\begin{aligned}
\omega_{\mu}^{\bar{i}} \delta_{\bar{l} \bar{l}} & =\delta_{\bar{i}}\left(e^{\bar{a} i}\left(\partial_{\bar{U}^{A}} e_{\bar{a}}^{\bar{l}}\right) \partial_{\mu} \bar{U}^{A}-e^{a \bar{\imath}}\left(\partial_{U^{A}} e_{a}^{i}\right) \partial_{\mu} U^{A}\right) \\
& =\partial_{U^{A}} K^{(U)} \partial_{\mu} U^{A}-\partial_{\bar{U}^{A}} K^{(U)} \partial_{\mu} \bar{U}^{A} .
\end{aligned}
$$

With this result at our disposal, we can again match the component action for the D3-brane with the standard supergravity expression. It is essential to notice that in the component action (2.36) the couplings of $\lambda$ and $\chi^{i}$ to $\partial_{\mu} \operatorname{Re}(T)$ and $\partial_{\mu} \operatorname{Re}\left(U^{A}\right)$ (via the spin connection term) have the same numerical coefficient. As a consequence, the complex structure sector and the Kähler sector have to couple to the chiral multiplets on the D3-brane in the same way. This observation leads us to propose the Kähler potential

$$
\begin{aligned}
K & =-\log [-\mathrm{i}(\tau-\bar{\tau})]-3 \log \left[-\mathrm{i}(T-\bar{T})\left(-\mathrm{i} \int \Omega \wedge \bar{\Omega}\right)^{\frac{1}{3}}+k(H, \bar{H})\right] \\
& =-\log [-\mathrm{i}(\tau-\bar{\tau})]-\log \left[-\mathrm{i} \int \Omega \wedge \bar{\Omega}\right]-3 \log \left[-\mathrm{i}(T-\bar{T})+\frac{k(H, \bar{H})}{\left(-\mathrm{i} \int \Omega \wedge \bar{\Omega}\right)^{\frac{1}{3}}}\right],
\end{aligned}
$$

which indeed produces the couplings to $\partial_{\mu} \operatorname{Im}(\tau)$ and $\partial_{\mu} \operatorname{Im}(T)$ as discussed above. In addition, it gives the correct coupling to $\chi^{i}$ via the standard supergravity terms,

$$
\begin{aligned}
\mathcal{L}^{\text {SUGRA }} \supset & -\delta_{i \bar{\jmath}} \bar{\chi}^{\bar{\jmath}} P_{R} \gamma^{\mu}\left(\partial_{\mu}-\frac{1}{4}\left[\partial_{\mu} U^{A} \partial_{U^{A}} K-\partial_{\mu} \bar{U}^{A} \partial_{\bar{U}^{A}} K\right]\right) \chi^{i} \\
& -\frac{1}{2} \delta_{i \bar{\jmath}} \bar{\jmath}^{\bar{\jmath}} P_{R} \gamma^{\mu} \Gamma_{k U^{A}}^{i} \partial_{\mu} U^{A} \chi^{k}-\frac{1}{2} \delta_{i \bar{\jmath}} \bar{\chi}^{i} P_{L} \gamma^{\mu} \Gamma_{\bar{k} \bar{U}^{A}}^{\bar{J}} \partial_{\mu} \bar{U}^{A} \chi^{\bar{k}},
\end{aligned}
$$

if we again drop terms involving $k(H, \bar{H})$ and its derivatives, while the couplings to $\lambda^{\prime}$ can be obtained from

$$
\mathcal{L}^{\text {SUGRA }} \supset-\frac{1}{2} \operatorname{Re}(f) \bar{\lambda}^{\prime} \gamma^{\mu}\left(\partial_{\mu}+\frac{1}{4}\left(\partial_{\mu} U^{A} \partial_{U^{A}} K-\partial_{\mu} \bar{U}^{A} \partial_{\bar{U}^{A}} K\right) \gamma_{*}\right) \lambda^{\prime}
$$

To conclude, notice that the maybe naively expected Kähler potential

$$
K=-\log [-\mathrm{i}(\tau-\bar{\tau})]-\log \left[-\mathrm{i} \int \Omega \wedge \bar{\Omega}\right]-3 \log [-\mathrm{i}(T-\bar{T})+k(H, \bar{H})]
$$

does not seem to reproduce the component action for the supersymmetric D3-brane. Instead, we need to use the Kähler potential given above in Eq. (2.46), which couples the world-volume scalars $H^{a}$ to the complex structure moduli $U^{A}$. Finally, in order to still reproduce the kinetic term for the $H^{a}, k(H, \bar{H})$ now needs to be chosen such that $\partial_{H^{a}} \partial_{\bar{H}^{\bar{b}}} k(H, \bar{H}) \approx \frac{1}{6} e^{e^{4}(\mathcal{A}+u)}\left(-\mathrm{i} \int \Omega \wedge \bar{\Omega}\right)^{\frac{1}{3}} g_{a \bar{b}}$.

This concludes our analysis of the (anti-)D3-brane action from the string theory perspective. In Sec. IV we will show how to obtain the anti-D3-brane action in Eq. (2.35) from $\mathcal{N}=1$ supergravity in four dimensions. For this purpose, nonlinear (local) supersymmetry will be employed in the language of constrained multiplets. We review the necessary ingredients in the following section.

\section{CONSTRAINED MULTIPLETS IN SUPERGRAVITY}

In this section we review some ingredients of nonlinear realizations and constrained multiplets in supergravity.
We focus on a particular set of constraints that we are going to employ in order to describe the anti-D3brane action [21,22]. It is important to keep in mind, however, that the choice of the required constraints in general is not unique, and different possibilities can occur. See, e.g., Refs. [23,27] for a discussion about this fact.

We use the conventions of Ref. [69], where 4D fermions are described by four-component Majorana spinors. In Appendix A 1, the relevant formulas are also given in the flat superspace language, following the conventions of Ref. [70].

\section{A. The Goldstino in a flat background}

When supersymmetry is spontaneously broken a Goldstino is present in the spectrum and transforms nonhomogeneously under supersymmetry transformations. A minimal action describing the Goldstino was proposed by Volkov and Akulov [39] and it is of the type 


$$
S_{\mathrm{VA}}=-M^{4} \int E^{0} \wedge E^{1} \wedge E^{2} \wedge E^{3}, \quad \text { with } \quad E^{\mu}=d x^{\mu}+\bar{\lambda} \gamma^{\mu} d \lambda
$$

where $\lambda$ is the spin- $1 / 2$ Goldstino and $M$ is a parameter of mass-dimension one, related to the supersymmetrybreaking scale. This action is invariant under the nonlinear transformation $(M=1)$

$$
\delta_{\epsilon} \lambda=\epsilon+\left(\bar{\lambda} \gamma^{\mu} \epsilon\right) \partial_{\mu} \lambda,
$$

which closes onto the $\mathcal{N}=1$ supersymmetry algebra.

We now briefly discuss how to reformulate the VolkovAkulov model in a language in which supersymmetry becomes manifest. When dealing with supersymmetric theories, it is convenient to embed fields into multiplets or superfields. A simple choice consists in identifying the Goldstino with the fermion $P_{L} \Omega$ of a chiral multiplet, ${ }^{9}$

$$
X=\left\{X, P_{L} \Omega, F\right\} .
$$

This multiplet, however, also contains a scalar $X$ which is not present in the Volkov-Akulov model (3.1). It is possible to eliminate this scalar in a supersymmetric way by imposing an additional constraint on the multiplet. If we require $X$ to be nilpotent [71-74], then the scalar in the lowest component becomes a function of the Goldstino and the auxiliary field $F$ :

$$
X^{2}=0 \Leftrightarrow X=\left\{\frac{\bar{\Omega} P_{L} \Omega}{2 F}, P_{L} \Omega, F\right\} .
$$

An invariant action for this nilpotent chiral multiplet is given by

$$
\begin{aligned}
S & =[X \bar{X}]_{D}+M^{2}[X]_{F} \\
& =\int d^{4} x\left(-\bar{\Omega} P_{L} \not \partial \Omega+\frac{\bar{\Omega} P_{L} \Omega}{2 F} \square \frac{\bar{\Omega} P_{R} \Omega}{2 \bar{F}}+F \bar{F}+M^{2}(F+\bar{F})\right) .
\end{aligned}
$$

Notice that the equations of motion of the auxiliary fields are modified with respect to the case in which supersymmetry is linearly realized, since the sgoldstino is replaced by a composite expression containing $F$. This is a general feature of models with nonlinearly realized supersymmetry and therefore attention has to be paid when going on shell. The equation of motion for the auxiliary field gives indeed

$$
F=-M^{2}-\frac{1}{4 M^{6}} \bar{\Omega} P_{R} \Omega \square\left(\bar{\Omega} P_{L} \Omega\right)+\frac{3}{16 M^{14}}\left(\bar{\Omega} P_{R} \Omega\right)\left(\bar{\Omega} P_{L} \Omega\right) \square\left(\bar{\Omega} P_{R} \Omega\right) \square\left(\bar{\Omega} P_{L} \Omega\right),
$$

and the on-shell action is

$$
S=\int d^{4} x\left(-M^{4}-\bar{\Omega} P_{L} \not \partial \Omega+\frac{1}{4 M^{4}} \bar{\Omega} P_{L} \Omega \square\left(\bar{\Omega} P_{R} \Omega\right)-\frac{1}{16 M^{12}}\left(\bar{\Omega} P_{R} \Omega\right)\left(\bar{\Omega} P_{L} \Omega\right) \square\left(\bar{\Omega} P_{R} \Omega\right) \square\left(\bar{\Omega} P_{L} \Omega\right)\right)
$$

By means of a field redefinition between $\lambda$ and $P_{L} \Omega$, one can prove that this action is equivalent to the VolkovAkulov model [75].

\section{B. Coupling the Goldstino to gravity}

Superconformal methods are very convenient when constructing supergravity actions. The strategy on which they rely consists in taking advantage of the full superconformal symmetry to fix all of the allowed interactions. This symmetry is then partially broken in order to

\footnotetext{
${ }^{9}$ We denote multiplets on which supersymmetry acts linearly with the same letter as their lowest components.
}

obtain Poincaré supergravity. With such a procedure it is possible to avoid field redefinitions, that might be needed to go to the Einstein frame when using other methods. Hence, in the present work we adopt the superconformal approach to supergravity, following the conventions of Ref. [69].

The superconformal action we are going to consider is of the type

$$
\begin{aligned}
S= & {\left[-3 X^{0} \bar{X}^{0} \mathrm{e}^{-K(X, \bar{X}) / 3}\right]_{D}+\left[\left(X^{0}\right)^{3} W(X)\right]_{F} } \\
& +\left[f_{A B}(X) \bar{\Lambda}^{A} P_{L} \Lambda^{B}\right]_{F},
\end{aligned}
$$

where $\left\{X^{I}\right\}, I=0, \ldots, n$ is a set of chiral multiplets where $X^{0}$ is the compensator, $\Lambda^{A}, A=1, \ldots n_{v}$ is a set of vector 
multiplets, $K$ is the Kähler potential, $W$ is the superpotential, and $f_{A B}$ is the gauge kinetic function. The compensator has Weyl weight 1 , while the other chiral multiplets have Weyl weight 0 . In order to obtain Poincaré supergravity one has to fix $X^{0}=\kappa^{-1} e^{\frac{K}{6}}$, which introduces the Planck scale into the theory.

A minimal model in which the Goldstino is coupled to gravity is given by

$$
K=X \bar{X}, \quad W=W_{0}+M^{2} X,
$$

where $X$ is the nilpotent Goldstino multiplet introduced before. In the case in which there are no vector multiplets, the action (3.8) reduces to

$$
\begin{aligned}
S= & {\left[-3 X^{0} \bar{X}^{0}+X^{0} \bar{X}^{0} X \bar{X}\right]_{D} } \\
& +\left[\left(X^{0}\right)^{3}\left(W_{0}+M^{2} X\right)\right]_{F}, \quad X^{2}=0 .
\end{aligned}
$$

This is the generalization of Eq. (3.5) to local supersymmetry and it has been studied in Refs. [76-80]. The model is sometimes called (pure) de Sitter supergravity because the only propagating modes are the graviton and the gravitino, the Goldstino being a pure gauge d.o.f. In addition, for certain values of the parameters in the scalar potential, the cosmological constant is positive. We stress again that, when calculating the component form of Eq. (3.10), it is important to substitute $X=\frac{\bar{\Omega} P_{L} \Omega}{2 F}$ before going on shell, since this will contribute to the equations of motion of the auxiliary fields.

\section{Other constrained multiplets}

A general procedure to constrain supersymmetric multiplets and remove any desired component has been given in Ref. [81]. In the following, besides the nilpotent Goldstino multiplet $X$, we are going to use other types of constrained multiplets, which we briefly review here. Notice that it is possible to implement them dynamically at the Lagrangian level, by means of a Lagrange multiplier [82]. In this way supersymmetry remains linear off shell.

\section{Constrained chiral multiplets $Y^{i}$}

Given a set of chiral multiplets $Y^{i}=\left\{Y^{i}, P_{L} \Omega^{i}, F^{i}\right\}$, by imposing the constraints $[83,84]$

$$
X^{2}=0, \quad X Y^{i}=0
$$

the scalar fields in the lowest components of $X$ and $Y^{i}$ are removed and expressed as

$$
X=\frac{\bar{\Omega} P_{L} \Omega}{2 F}, \quad Y^{i}=\frac{\bar{\Omega}^{i} P_{L} \Omega}{F}-\frac{\bar{\Omega} P_{L} \Omega}{2 F^{2}} F^{i} .
$$

Therefore, these multiplets only contain fermions as propagating d.o.f. They have been used in
Refs. [21,22] to describe the world-volume spinors of an anti-D3-brane.

\section{Constrained chiral multiplets $\boldsymbol{H}^{a}$}

Given another set of chiral multiplets $H^{a}=\left\{H^{a}\right.$, $\left.P_{L} \Omega^{a}, F^{a}\right\}$, by imposing the constraints [74]

$$
X^{2}=0, \quad X \bar{H}^{a}=\text { chiral }
$$

the fermion and the auxiliary field in $H^{a}$ are removed and expressed as

$$
\begin{gathered}
P_{L} \Omega^{a}=\frac{\not D H^{a}}{\bar{F}} P_{R} \Omega, \\
F^{a}=\mathcal{D}_{\mu}\left(\frac{\bar{\Omega}}{\bar{F}}\right) \gamma^{\nu} \gamma^{\mu}\left(\frac{P_{R} \Omega}{\bar{F}}\right) \mathcal{D}_{\nu} H^{a}+\frac{\bar{\Omega} P_{R} \Omega}{2 \bar{F}^{2}} \square H^{a} .
\end{gathered}
$$

Therefore, the chiral multiplets $H^{a}$ only contain a complex scalar as an independent component field. Notice that, due to this fact, a superpotential of the type $W=W(H)$ does not lead to mass terms for the scalars $H^{a}$, but to fermionic terms containing Goldstino interactions.

\section{Constrained chiral field strength multiplet $\boldsymbol{P}_{L} \boldsymbol{\Lambda}_{\alpha}$}

The field strength chiral multiplet that has the gaugino as its lowest component is

$$
P_{L} \Lambda_{\alpha}=\left\{P_{L} \Lambda_{\alpha},\left(P_{L} \chi\right)_{\beta \alpha}, F_{\alpha}^{\Lambda}\right\}
$$

where

$$
\begin{gathered}
\left(P_{L} \chi\right)_{\beta \alpha}=\sqrt{2}\left[-\frac{1}{4}\left(P_{L} \gamma^{a b} C\right)_{\beta \alpha} \hat{F}_{a b}+\frac{i}{2} \mathrm{D}\left(P_{L} C\right)_{\beta \alpha}\right], \\
F_{\alpha}^{\Lambda}=\left(\not D P_{R} \Lambda\right)_{\alpha},
\end{gathered}
$$

and where we have explicitly written the spinorial indices to avoid confusion. $C_{\alpha \beta}$, which satisfies $C^{T}=-C$, is the matrix used to raise and lower fermionic indices, while $\hat{F}_{a b}=e_{a}^{\mu} e_{b}^{\nu}\left(2 \partial_{[\mu} A_{\nu]}+\bar{\psi}_{[\mu} \gamma_{\nu]} \lambda\right)$ is the covariant vector field strength and $\mathrm{D}$ is the real auxiliary field. This multiplet is the analog of the superfield strength $W_{\alpha}=-\frac{1}{4} \bar{D}^{2} D_{\alpha} V$ defined in superspace, which indeed is chiral and has the gaugino in the lowest component.

The gaugino can be eliminated by imposing the constraint

$$
X P_{L} \Lambda_{\alpha}=0
$$

which gives 


$$
\begin{aligned}
P_{L} \Lambda_{\alpha}= & \frac{1}{F}\left(\bar{\Omega} P_{L} \chi\right)_{\alpha}-\frac{X}{F} \not D_{\alpha}{ }^{\beta}\left(\frac{\left(\bar{\Omega} P_{R} \chi\right)_{\beta}}{\bar{F}}\right) \\
& +\frac{X}{F} \not D_{\alpha}{ }^{\beta}\left(\frac{\bar{X}}{\bar{F}} \not D_{\beta}{ }^{\gamma}\left(\frac{\left(\bar{\Omega} P_{L} \chi\right)_{\gamma}}{F}\right)\right) \\
& -\frac{X \bar{X}}{F^{2} \bar{F}^{2}}(\not D \not D X)_{\alpha}{ }^{\beta}\left(\gamma^{\mu}\right)_{\beta}{ }^{\delta}\left(\mathcal{D}_{\mu} \bar{\Omega} P_{R} \chi\right)_{\delta},
\end{aligned}
$$

where $X=\frac{\bar{\Omega} P_{L} \Omega}{2 F}$ and $\left(\bar{\Omega} P_{L} \chi\right)_{\alpha}=\Omega^{\beta}\left(P_{L}\right)_{\beta}^{\gamma} \chi_{\gamma \alpha}$. Therefore, the constrained multiplet $P_{L} \Lambda_{\alpha}$ describes only an Abelian gauge vector as an independent propagating d.o.f. The superspace constraint corresponding to Eq. (3.19) is $X W_{\alpha}=0$.

\section{CONSTRUCTING A SUPERGRAVITY ACTION FOR THE ANTI-D3-BRANE}

In this section we recast the anti-D3-brane action (2.35) in the language of $\mathcal{N}=1$ supergravity in four dimensions. The rewriting of the fermionic action coupled to the closedstring moduli was done already in Ref. [27] [see, e.g., their Eqs. (3.51) and (3.52)]. ${ }^{10}$ Here, we extend this result by also considering the bosonic part, together with the terms that mix world-volume bosons and fermions, and the sector containing the $\mathrm{U}(1)$ gauge vector.

The logic consists in embedding each of the worldvolume fields into one of the constrained multiplets presented in Sec. III. In this way we will be able to use the standard language of supergravity - namely, to write down a Kähler potential, a superpotential, and a gauge kinetic function-but at the same time the nonlinear realization of supersymmetry will be manifest. The very fact that it is possible to use nonlinear supersymmetry to rewrite the anti-D3-brane action confirms that the antibrane spontaneously breaks supersymmetry.

Note that branes break supersymmetry generically at the string scale. In our case, the anti-D3-brane sits at the bottom of a warped throat and therefore the string scale is warped down compared to the bulk string scale. A recent discussion of these scales can be found, e.g., in Ref. [44]. The warpeddown string scale, in our conventions with $l_{s}=$ $2 \pi \sqrt{\alpha^{\prime}}=1$, is given by the first term in Eq. (2.35): $M_{s}^{4}=2 e^{4 \mathcal{A}-8 u}$. This sets the supersymmetry-breaking scale, as can be seen by looking, e.g., at Eqs. (3.6) and (3.7) above. One expects that at this scale linear supersymmetry will be restored, and indeed massive open-string states arise as new d.o.f. The particular Klebanov-Strassler throat geometry [62], which has been intensively studied in the KKLT context, has a three-sphere at the bottom of the warped throat. Anti-D3-branes at the bottom of the throat

\footnotetext{
${ }^{10}$ We thank Flavio Tonioni and the authors of Ref. [27] for alerting us to a problem with their mass term for the fermions. We will rectify this and present below two different ways of writing the fermionic mass term.
}

can then decay via the nucleation of an NS5-brane that is wrapping an $S^{2}$ inside the $S^{3}$ [5]. Such a decay leads to a supersymmetric state and one can actually write down a supergravity theory with linear supersymmetry by including the infinite tower of Kaluza-Klein (KK) modes associated with the $S^{3}$ [31]. Therefore, in this particular case one finds that new states come in already below the supersymmetry-breaking scale and lead to a restoration of linear supersymmetry. Having the supersymmetrybreaking scale at the warped-down string scale, which is above the warped-down KK scale at which the fourdimensional effective field theory breaks down, might seem worrisome. However, the hallmark of a supergravity theory is the presence of a gravitino and the mass of the gravitino in the KKLT scenario can be well below the KK scale. Thus, a description in terms of a four-dimensional $\mathcal{N}=1$ theory is appropriate.

\section{A. Goldstino and matter component fields}

We start by considering the couplings involving scalars and fermions, while we will focus on the gauge vector in Sec. IV B. In particular, we generate the mass terms for the fermions with a different mechanism with respect to Ref. [27] and we show how to also include the anti-D3brane world-volume scalars $H^{a}$ in the supergravity action. An alternative way of producing a fermionic mass term is presented in Sec. IV C.

The first step is to embed the Goldstino $\lambda$ and the triplet of fermions $\chi^{i}$ into, respectively, a chiral multiplet $X$ and a triplet of chiral multiplets $Y^{i}$ satisfying the constraints (3.11). The kinetic terms of the spin- $1 / 2$ fields in Eq. (2.35) can then be generated from the following Kähler potential, in which the bulk moduli are coupled to world-volume fermions [27]:

$$
\begin{aligned}
K= & -\log (-\mathrm{i}(\tau-\bar{\tau}))-3 \log \left[(-\mathrm{i}(T-\bar{T})) f\left(U^{A}, \bar{U}^{A}\right)^{\frac{1}{3}}\right] \\
& -3 \log \left(1-\frac{e^{-4 \mathcal{A}} X \bar{X}}{3(-\mathrm{i}(\tau-\bar{\tau}))(-\mathrm{i}(T-\bar{T})) f\left(U^{A}, \bar{U}^{A}\right)}\right. \\
& \left.-\frac{e^{-4 \mathcal{A}} \delta_{i \bar{\jmath}} Y^{i} \bar{Y}^{\bar{j}}}{3(-\mathrm{i}(\tau-\bar{\tau}))(-\mathrm{i}(T-\bar{T}))^{2} f\left(U^{A}, \bar{U}^{A}\right)^{\frac{1}{3}}}\right) .
\end{aligned}
$$

From now on we use $f\left(U^{A}, \bar{U}^{A}\right)=-\mathrm{i} \int \Omega \wedge \bar{\Omega}$ to avoid confusion between the holomorphic $(3,0)$-form and the fermions $\Omega, \Omega^{i}$. The couplings of $X$ and $Y^{i}$ to the bulk moduli are fixed as follows. The coupling to $\tau$ is determined by requiring modular invariance for the world-volume action (see Sec. VA below for details). For what concerns the other moduli, the couplings to $X$ are fixed by matching with the scalar potential in Eq. (2.35), while those to $Y^{i}$ are fixed by matching with the Kähler covariant kinetic terms of the massive spin- $1 / 2$ world-volume spinors (cf. Sec. IIC). In particular, the fermions $\Omega^{i}$ inside $Y^{i}$ are related to $\chi^{i}$ by the field redefinitions 


$$
P_{L} \Omega^{i}=2 \mathrm{i} e^{4 \mathcal{A}-\frac{\phi}{2}} f\left(U^{A}, \bar{U}^{A}\right)^{\frac{1}{6}} P_{L} \chi^{i}+\ldots,
$$

where dots stand for higher-order terms. Notice that we are not matching the supergravity expression with the kinetic term of $\lambda$ in Eq. (2.35) since, due to the fact that such a fermion is a Goldstino, its couplings are not physical and they can be set to zero by going to the unitary gauge. For what follows, it is sufficient to keep in mind that, in our supergravity description, the Goldstino resides in the multiplet $X$, namely, $P_{L} \Omega \sim P_{L} \lambda+\cdots$, where dots stand for higher-order terms. We stress that the presence of the Goldstino is an essential feature of the anti-D3-brane and a similar reasoning cannot be repeated in the case of the D3-brane.

The superpotential that sources the supersymmetry breaking and gives rise to the anti-D3-brane uplift term is

$$
W=W_{\mathrm{GVW}}+W_{n p}+M^{2} X,
$$

where $M^{2}=\sqrt{2}$. The parameter $M$ is related to the supersymmetry-breaking scale, which is the warped-down string scale. By rescaling $X$ we have included the warp factor in the Kähler potential and we have set the anti-D3brane tension to $T_{D 3}=2 \pi=M^{4} \pi$. The very form of the superpotential (4.3) implies that supersymmetry is spontaneously broken by the auxiliary field of $X$ and therefore it is consistent to identify the Goldstino $\lambda$ with the fermion $\Omega$ inside $X$, at leading order.

The Kähler potential and superpotential presented so far correctly reproduce the kinetic terms for the fermions and the scalar potential in Eq. (2.35). On the other hand, at this stage the fermions are massless in the supergravity theory, since none of the couplings we introduced produce a mass term for them. In order to give a mass to the fermion triplet, it was proposed in Refs. [21,27] to add a contribution $W_{m}=h_{i j} Y^{i} Y^{j}$ to the superpotential. However, when the axiodilaton is dynamical and not integrated out, then such a mass term would require $h_{i j} \propto \bar{G}_{3}$ to be antiholomorphic in $\tau$, which seems incompatible with supersymmetry. Instead of adding a term to the superpotential, one can therefore follow a different strategy and modify the Kähler potential. The required modification of $K$ given in Eq. (4.1) and that generates the desired fermionic mass term is

$$
\begin{aligned}
K= & -\log (-\mathrm{i}(\tau-\bar{\tau}))-3 \log \left[(-\mathrm{i}(T-\bar{T})) f\left(U^{A}, \bar{U}^{A}\right)^{\frac{1}{3}}\right] \\
& -3 \log \left(1-\frac{e^{-4 \mathcal{A}} X \bar{X}}{3(-\mathrm{i}(\tau-\bar{\tau}))(-\mathrm{i}(T-\bar{T})) f\left(U^{A}, \bar{U}^{A}\right)}-\frac{e^{-4 \mathcal{A}} \delta_{i \bar{\jmath}} Y^{i} \bar{Y}^{\bar{j}}}{3(-\mathrm{i}(\tau-\bar{\tau}))(-\mathrm{i}(T-\bar{T}))^{2} f\left(U^{A}, \bar{U}^{A}\right)^{\frac{1}{3}}}\right. \\
& \left.+\frac{e^{-8 \mathcal{A}}\left(m_{i j} \bar{X} Y^{i} Y^{j}+\bar{m}_{\bar{l} \bar{\jmath}} X \bar{Y}^{\bar{\imath}} \bar{Y}^{\bar{j}}\right)}{6 M^{2}(-\mathrm{i}(\tau-\bar{\tau}))^{\frac{3}{2}}(-\mathrm{i}(T-\bar{T}))^{\frac{3}{2}} f\left(U^{A}, \bar{U}^{A}\right)^{\frac{5}{6}}}\right)
\end{aligned}
$$

where $m_{i j}$ is given in Eq. (2.33) above. As we discuss more extensively in Sec. VA, this modification of the Kähler potential does not spoil the modular invariance of the supergravity action. In Sec. IV C we present an alternative mechanism, in which the mass to the fermion triplet is given by a superpotential term. However, such a construction requires some technical explanation in order to be presented properly and this is the reason why we postpone it for the time being.

Now we present how to introduce the dependence on the world-volume scalars $H^{a}$ parametrizing small fluctuations around the position of the anti-D3-brane in the warped throat. The kinetic term of $H^{a}$ arises from the DBI action, which is the same for D3-branes and anti-D3-branes. It is known to be well described by the Kähler potential in Eq. (2.7) in the case of fixed complex structure moduli and we have shown that it should be modified as in Eq. (2.46) when the complex structure and axiodilaton are dynamical. We can now consider a simple prescription to generalize the supergravity Kähler potential (4.4). We first embed the scalar fields into the constrained multiplets $H^{a}$ introduced in Sec. III C 2, that contain only a scalar in the lowest component as an independent d.o.f. Then we let $\mathcal{A}$ depend generically on $H^{a}$ and we formally shift the volume modulus as $-\mathrm{i}(T-\bar{T}) \rightarrow-\mathrm{i}(T-\bar{T})+k\left(H^{a}, \bar{H}^{a}\right) / f\left(U^{A}, \bar{U}^{A}\right)^{\frac{1}{3}}=e^{4 u}$ to match the results. As a result, we get the following Kähler potential of $\mathcal{N}=1$ supergravity in four dimensions:

$$
\begin{aligned}
K= & -\log (-\mathrm{i}(\tau-\bar{\tau}))-3 \log \left[(-\mathrm{i}(T-\bar{T})) f\left(U^{A}, \bar{U}^{A}\right)^{\frac{1}{3}}+k\left(H^{a}, \bar{H}^{a}\right)\right] \\
& -3 \log \left(1-\frac{e^{-4 \mathcal{A}\left(H^{a}, \bar{H}^{a}\right)-4 u}}{3(-\mathrm{i}(\tau-\bar{\tau})) f\left(U^{A}, \bar{U}^{A}\right)} X \bar{X}-\frac{e^{-4 \mathcal{A}\left(H^{a}, \bar{H}^{a}\right)-8 u}}{3(-\mathrm{i}(\tau-\bar{\tau})) f\left(U^{A}, \bar{U}^{A}\right)^{\frac{1}{3}}} \delta_{i \bar{\jmath}} Y^{i} \bar{Y}^{\bar{\jmath}}+\frac{e^{-8 \mathcal{A}\left(H^{a}, \bar{H}^{a}\right)-6 u}\left(m_{i j} \bar{X} Y^{i} Y^{j}+\bar{m}_{\bar{\imath} \bar{\jmath}} X \bar{Y}^{\bar{\imath}} \bar{Y}^{\bar{\jmath}}\right)}{6 M^{2}(-\mathrm{i}(\tau-\bar{\tau}))^{\frac{3}{2}} f\left(U^{A}, \bar{U}^{A}\right)^{\frac{5}{6}}}\right) .
\end{aligned}
$$


As a consequence of this last step, the superpotential $W_{n p}$ also gets a dependence on the scalars $H^{a}$. However, as we explained previously, the net result of this additional dependence would be to produce corrections which are highly suppressed in the regime we are considering. For all of the purposes of the present work, we can therefore neglect the dependence of $W_{n p}$ on $H^{a}$ and keep considering Eq. (4.3) as the expression for the superpotential.

Having identified the Kähler potential and the superpotential, we can use the rules of $\mathcal{N}=1$ supergravity to calculate the scalar potential. The result is of the form

$$
V=V_{K K L T}+V_{\overline{D 3}}
$$

where $V_{K K L T}$ contains the contributions from the supergravity bulk fields, while $V_{\overline{D 3}}$ is the uplift term coming from the anti-D3-brane,

$$
\begin{aligned}
V_{\overline{D 3}} & =\frac{M^{4} e^{4 \mathcal{A}\left(H^{a}, \bar{H}^{a}\right)}}{\left(-\mathrm{i}(T-\bar{T})+k\left(H^{a}, \bar{H}^{a}\right) f\left(U^{A}, \bar{U}^{A}\right)^{-\frac{1}{3}}\right)^{2}} \\
& =2 e^{4 \mathcal{A}\left(H^{a}, \bar{H}^{a}\right)-8 u},
\end{aligned}
$$

which reproduces Eq. (2.12), as desired. We recall that to calculate the scalar potential with the constrained multiplets
$X$ and $Y^{i}$, it is sufficient to perform the calculation in the usual manner and then set $X=Y^{i}=0$ in the final result.

\section{B. Gauge vector field and theta term}

We now focus on the part of the action containing the world-volume gauge vector field. As we mentioned in Sec. II A, the kinetic term originating from the DBI action is the same as that of the D3-brane vector, whereas the CS term has the opposite sign due to the difference in the RR charge. As a consequence of this sign flip, it seems that the gauge kinetic function needs to depend on an antichiral multiplet, $\bar{f}=\bar{f}(\bar{\tau})$, and this would be an obstruction to rephrasing the antibrane vector field into an $\mathcal{N}=1$ supersymmetric language. However, as we are going to show, the fact that supersymmetry is spontaneously broken and nonlinearly realized allows us to also correctly embed the CS term. In this subsection, therefore, we show explicitly how to describe such a CS term with the appropriate sign for the anti-D3-brane case.

As a first step, we embed the world-volume vector field into a chiral field strength multiplet $P_{L} \Lambda_{\alpha}$ and we constrain it as in Eq. (3.19). As explained before, this constraint removes the gaugino, leaving only the $\mathrm{U}(1)$ vector as the independent physical d.o.f. If we then consider the standard supergravity action for the vector multiplet [69], ${ }^{11}$

$$
-\frac{1}{4}\left[f(\tau) \bar{\Lambda} P_{L} \Lambda\right]_{F}=\int d^{4} x \sqrt{-g_{4}}\left(-\frac{\operatorname{Re}(f)}{4} F_{\mu \nu} F^{\mu \nu}+\frac{\operatorname{Im}(f)}{8} \frac{\epsilon^{\mu \nu \rho \sigma}}{\sqrt{-g_{4}}} F_{\mu \nu} F_{\rho \sigma}+\frac{\operatorname{Re}(f)}{2} \mathrm{D}^{2}+\ldots\right),
$$

with $f(\tau)=-\mathrm{i} \tau$ and where the dots stand for fermionic terms, we notice that the term proportional to $\operatorname{Im}(f)=$ $-\operatorname{Re}(\tau)$ has the opposite sign compared to Eq. (2.35). We would therefore like to flip this sign by subtracting from the action twice the same contribution. In order to perform this step in a supersymmetric way, we construct a deformation of the action which is similar in spirit to the recently proposed new Fayet-Iliopoulos $D$-terms in supergravity [28,32,85-90]. In Ref. [28] it was shown how to deform Eq. (4.8) and introduce a coupling linear in the auxiliary field $D$, without spoiling the gauge invariance and without requiring the gauging of the $R$ symmetry, which is needed for the standard Fayet-Iliopoulos $D$-term. This new coupling shifts the vacuum expectation value of $D$ and, as a consequence, supersymmetry is spontaneously broken by a $D$-term. In the same spirit, we would like to introduce a coupling $-\frac{1}{4} \operatorname{Im}(f) \epsilon^{\mu \nu \rho \sigma} F_{\mu \nu} F_{\rho \sigma}$, together with all of the additional interactions required by supersymmetry, in order to flip the sign in the theta term in Eq. (4.8). In the following we directly give the expression of such a

\footnotetext{
${ }^{11}$ In Ref. [69] the overall factor $-\frac{1}{4}$ was understood, but we prefer to keep it explicit for convenience.
}

deformation, but more details about its derivation and new $D$-terms in general are given in Appendix B.

Given a constrained field strength multiplet $P_{L} \Lambda_{\alpha}$, in order to reproduce the vector multiplet interactions of Eq. (2.35), we propose the following supergravity action:

$$
\begin{aligned}
S_{V}= & -\frac{1}{4}\left[f(\tau) \bar{\Lambda} P_{L} \Lambda\right]_{F} \\
& +\left[\frac{X \bar{X}\left(X^{0} \bar{X}^{0} e^{-\frac{K}{3}}\right)^{3}}{\Sigma\left(\bar{X}^{0} e^{-\frac{K}{6}} \bar{X}\right) \bar{\Sigma}\left(X^{0} e^{-\frac{K}{6}} X\right)} \operatorname{Im}(f) \operatorname{Im}\left(\Sigma\left(\bar{\omega}^{2}\right)\right)\right]_{D},
\end{aligned}
$$

where we defined the multiplets

$$
\omega^{2}=\frac{\bar{\Lambda} P_{L} \Lambda}{\left(X^{0} \bar{X}^{0} e^{-\frac{K}{3}}\right)^{2}}, \quad \bar{\omega}^{2}=\frac{\bar{\Lambda} P_{R} \Lambda}{\left(X^{0} \bar{X}^{0} e^{-\frac{K}{3}}\right)^{2}},
$$

and where $(\bar{\Sigma}) \Sigma$ is the (anti)chiral projector in the superconformal setup. We always assume that $X$ is nilpotent. This action is made up of the standard kinetic coupling for the vector multiplet (4.8) and a second, novel term, whose origin is presented in Appendix B. The property of this coupling that is important for the present discussion is that 
its component expansion starts precisely with the desired theta term,

$$
\begin{aligned}
S_{\theta \text {-term }} & =\left[\frac{X \bar{X}\left(X^{0} \bar{X}^{0} e^{-\frac{K}{3}}\right)^{3}}{\Sigma\left(\bar{X}^{0} e^{-\frac{K}{6}} \bar{X}\right) \bar{\Sigma}\left(X^{0} e^{-\frac{K}{6}} X\right)} \operatorname{Im}(f) \operatorname{Im}\left(\Sigma\left(\bar{\omega}^{2}\right)\right)\right]_{D} \\
& =-\frac{1}{4} \int d^{4} x \operatorname{Im}(f) \epsilon^{\mu \nu \rho \sigma} F_{\mu \nu} F_{\rho \sigma}+\ldots
\end{aligned}
$$

where the dots stand for fermionic terms. After fixing the superconformal symmetry with $X^{0}=\kappa^{-1} e^{\frac{K}{6}}$, the bosonic sector of Eq. (4.9) reduces to the desired result $(\kappa=1)$

$$
\begin{aligned}
S_{V, \mathrm{bos}}= & \int d^{4} x \sqrt{-g_{4}}\left[-\frac{1}{4} \operatorname{Im}(\tau) F^{\mu \nu} F_{\mu \nu}\right. \\
& \left.+\frac{1}{8} \operatorname{Re}(\tau) \frac{\epsilon^{\mu \nu \rho \sigma}}{\sqrt{-g_{4}}} F_{\mu \nu} F_{\rho \sigma}\right],
\end{aligned}
$$

where we used $f(\tau)=-\mathrm{i} \tau$ and we integrated out the auxiliary field $D$. In particular, the sign of the theta term has been flipped since the contribution coming from the second term in Eq. (4.9) is minus twice that arising from the first term.

A few comments are in order at this point. We find that Eq. (4.9) correctly realizes the bosonic part of the worldvolume vector action (2.11). It is worth noting that the second term in Eq. (4.9), which is essential in order to realize the correct CS term, can be consistently introduced only if the auxiliary field $F$ of $X$ is nonvanishing. This condition is always satisfied within our setup, since the anti-D3-brane breaks supersymmetry spontaneously and the fermion in $X$ provides the Goldstino. Notice also that the coupling (4.11) contains terms quadratic (and also of higher order) in the fermions in its component expansion, which might jeopardize the matching of the anti-D3-brane action with our supergravity proposal. However, due to the nonlinear realization of supersymmetry, the fermionic terms will be functions of the Goldstino and vanish identically in the unitary gauge. Therefore, even if differences might be present in the fermionic couplings between the anti-D3-brane action and the supergravity one, these differences are not physical and can be removed by an appropriate gauge choice. We stress that this is true as long as the fermion in $X$ is completely aligned with the Goldstino.

By exploiting the properties of the operator $\Sigma$ and the constraints (3.4) and (3.19), it is possible to recast the action (4.9) into a more suggestive form. Notice first that

$$
\begin{aligned}
{\left[f(\tau) \bar{\Lambda} P_{L} \Lambda\right]_{F} } & =\left[\frac{\Sigma\left(\bar{X}^{0} e^{-\frac{K}{6}} \bar{X} f(\tau)\right)}{\Sigma\left(\bar{X}^{0} e^{-\frac{K}{6}} \bar{X}\right)} \bar{\Lambda} P_{L} \Lambda\right]_{F} \\
& =\left[\Sigma\left(\frac{\bar{X}^{0} e^{-\frac{K}{6}} \bar{X} f(\tau)}{\Sigma\left(\bar{X}^{0} e^{-\frac{K}{6}} \bar{X}\right)}\right) \bar{\Lambda} P_{L} \Lambda\right]_{F}
\end{aligned}
$$

where we used the fact that $\Sigma(A B)=A \Sigma(B)$ if $A$ is chiral and $B$ has weights $(w, w-2)$, as proved in Ref. [82]. Then, since $P_{L} \Lambda_{\alpha}$ is constrained, we have

$$
\begin{aligned}
S_{\theta-\text { term }} & =\left[\frac{X \bar{X}\left(X^{0} \bar{X}^{0} e^{-\frac{K}{3}}\right)^{3}}{\Sigma\left(\bar{X}^{0} e^{-\frac{K}{6}} \bar{X}\right) \bar{\Sigma}\left(X^{0} e^{-\frac{K}{6}} X\right)} \operatorname{Im}(f) \operatorname{Im}\left(\Sigma\left(\bar{\omega}^{2}\right)\right)\right]_{D} \\
& =\left[\frac{\mathrm{i}}{2} \frac{\bar{X}^{0} e^{-\frac{K}{6}} \bar{X}}{\bar{\Sigma}\left(X^{0} e^{-\frac{K}{6}} X\right)} \operatorname{Im}(f) \bar{\Lambda} P_{L} \Lambda+\text { c.c. }\right]_{D} \\
& =\left[\frac{\mathrm{i}}{2} \Sigma\left(\frac{\bar{X}^{0} e^{-\frac{K}{6}} \bar{X}}{\bar{\Sigma}\left(X^{0} e^{-\frac{K}{6}} X\right)} \operatorname{Im}(f)\right) \bar{\Lambda} P_{L} \Lambda\right]_{F}
\end{aligned}
$$

where in going from the second to the third line we used the fact that $[C]_{D}=\frac{1}{2}[\Sigma(C)]_{F}$, another property stated, e.g., in Ref. [82]. Using these results, the two terms in Eq. (4.9) can be put together and the vector multiplet action acquires the more familiar form

$$
\begin{aligned}
S_{V} & =-\frac{1}{4}\left[\Sigma\left(\frac{\bar{X}^{0} e^{-\frac{K}{6}} \bar{X} \bar{f}(\bar{\tau})}{\Sigma\left(\bar{X}^{0} e^{-\frac{K}{6}} \bar{X}\right)}\right) \bar{\Lambda} P_{L} \Lambda\right]_{F} \\
& \equiv-\frac{1}{4}\left[\hat{f}_{\overline{D 3}}(\bar{\tau}, \bar{X}) \bar{\Lambda} P_{L} \Lambda\right]_{F},
\end{aligned}
$$

with $\bar{f}=\operatorname{Re}(f)-\operatorname{iIm}(f)$ and where we defined the composite (or generalized) anti-D3-brane gauge kinetic function

$$
\hat{f}_{\overline{D 3}}=\Sigma\left(\frac{\left(\bar{X}^{0} e^{-\frac{K}{6}} \bar{X}\right) \bar{f}(\bar{\tau})}{\Sigma\left(\bar{X}^{0} e^{-\frac{K}{6}} \bar{X}\right)}\right) .
$$

This is a chiral multiplet that is antiholomorphic in $\tau$. It is important to notice that $\hat{f}_{\overline{D 3}}$ contains Goldstino interactions, which implement the nonlinear realization of supersymmetry and are essential in order to consistently couple the vector multiplet to $\bar{\tau}$. The lowest component of $\hat{f}_{\overline{D 3}}$ is given by

$$
\hat{f}_{\overline{D 3}}=\bar{f}(\bar{\tau})+\text { fermions }
$$

and the fermionic terms vanish identically in the unitary gauge in which the Goldstino is set to zero. Indeed, one can equally think of $\hat{f}_{\overline{D 3}}$ as a chiral multiplet that satisfies the additional constraint

$$
X \bar{X} \hat{f}_{\overline{D 3}}=X \bar{X} \bar{f}(\bar{\tau})
$$

\section{Superpotential mass for the triplet of fermions}

In the previous section we have seen how nonlinear supersymmetry allows us to consistently couple $\bar{f}(\bar{\tau})=\mathrm{i} \bar{\tau}$ to the vector field, using a manifestly supersymmetric language. This strategy is indeed general and can also be employed to describe the mass term of the fermion triplet. 
In fact, we recall that the obstruction to producing such a term from the superpotential was that, in the case of the anti-D3-brane, it depends on $\bar{\tau}$ instead of $\tau$.

Inspired by the composite anti-D3-brane gauge kinetic function $\hat{f}_{\overline{D 3}}$ introduced before, we define the chiral multiplet

$\hat{M}_{i j}=\Sigma\left(\frac{\left(\bar{X}^{0} e^{-\frac{K}{6}} \bar{X}\right) e^{-4 \mathcal{A}-2 u} m_{i j}}{\Sigma\left(\bar{X}^{0} e^{-\frac{K}{6}} \bar{X}\right)(-\mathrm{i}(\tau-\bar{\tau}))^{\frac{1}{2}} f\left(U^{A}, \bar{U}^{A}\right)^{-\frac{1}{6}}}\right)$,

where $m_{i j}$ is the fermion mass given in Eq. (2.32). The lowest component of this multiplet is

$\hat{M}_{i j}=\frac{e^{-4 \mathcal{A}-2 u}}{(-\mathrm{i}(\tau-\bar{\tau}))^{\frac{1}{2}} f\left(U^{A}, \bar{U}^{A}\right)^{-\frac{1}{6}}} m_{i j}+$ fermions

and the fermionic terms vanish in the unitary gauge. Indeed, $\hat{M}_{i j}$ satisfies the constraint

$$
X \bar{X} \hat{M}_{i j}=X \bar{X}\left(\frac{e^{-4 \mathcal{A}-2 u} m_{i j}}{(-\mathrm{i}(\tau-\bar{\tau}))^{\frac{1}{2}} f\left(U^{A}, \bar{U}^{A}\right)^{-\frac{1}{6}}}\right) .
$$

A mass for the fermions in $Y^{i}$ can then be produced by a superpotential holomorphic in $\hat{M}_{i j}$ and $Y^{i}$ of the type

$$
W_{m}(\hat{M}, Y)=\frac{1}{2} \hat{M}_{i j} Y^{i} Y^{j} .
$$

We stress that this type of constructions, namely, $\hat{f}_{\overline{D 3}}$ and $\hat{M}_{i j}$, can be consistently defined only in a setup in which supersymmetry is spontaneously broken and the auxiliary field of $X$ acquires a nonvanishing vacuum expectation value. $^{12}$

\section{SUMMARY: THE SUPERGRAVITY ACTION AND ITS MODULAR INVARIANCE}

In this section we summarize our results and present some consistency checks on the expected modular properties of the anti-D3-brane action. We have showed that, up to quadratic terms in the fermions, the anti-D3-brane action (2.35) can be described in $\mathcal{N}=1$ supergravity in four dimensions by

$S=\left[\hat{f}_{\overline{D 3}}(\bar{\tau}, \bar{X}) \bar{\Lambda} P_{L} \Lambda\right]_{F}+\left[-3 X^{0} \bar{X}^{0} e^{-\frac{K}{3}}\right]_{D}+\left[\left(X^{0}\right)^{3} W\right]_{F}$.

The anti-D3-brane gauge kinetic function is built out of the Goldstino and the axiodilaton and is defined as the chiral multiplet

$$
\hat{f}_{\overline{D 3}}=\Sigma\left(\frac{\bar{X}^{0} e^{-\frac{K}{6}} \bar{X} \bar{f}(\bar{\tau})}{\Sigma\left(\bar{X}^{0} e^{-\frac{K}{6}} \bar{X}\right)}\right)
$$

with $\bar{f}(\bar{\tau})=\mathrm{i} \bar{\tau}$. We presented two different expressions for the Kähler potential and the superpotential, depending on how the mass term for the fermions in $Y^{i}$ is generated. They lead to the same physical action and are related by field redefinitions involving the Goldstino. One possibility is to generate the mass for the triplet of fermions from the Kähler potential

$$
\begin{aligned}
K_{1}= & -\log (-\mathrm{i}(\tau-\bar{\tau}))-3 \log \left[(-\mathrm{i}(T-\bar{T})) f\left(U^{A}, \bar{U}^{A}\right)^{\frac{1}{3}}+k\left(H^{a}, \bar{H}^{a}\right)\right] \\
& -3 \log \left(1-a X \bar{X}-b \delta_{i \bar{\jmath}} Y^{i} \bar{Y}^{\bar{j}}+c\left(m_{i j} \bar{X} Y^{i} Y^{j}+\bar{m}_{\bar{\imath} \bar{\jmath}} X \bar{Y}^{\bar{l}} \bar{Y}^{\bar{\jmath}}\right)\right),
\end{aligned}
$$

where $f\left(U^{A}, \bar{U}^{A}\right)=-\mathrm{i} \int \Omega \wedge \bar{\Omega}$ and

$$
\begin{gathered}
a=\frac{e^{-4 \mathcal{A}\left(H^{a}, \bar{H}^{a}\right)}}{3(-\mathrm{i}(\tau-\bar{\tau}))\left(-\mathrm{i}(T-\bar{T})+k\left(H^{a}, \bar{H}^{a}\right) f\left(U^{A}, \bar{U}^{A}\right)^{-\frac{1}{3}}\right) f\left(U^{A}, \bar{U}^{A}\right)}, \\
b=\frac{e^{-4 \mathcal{A}\left(H^{a}, \bar{H}^{a}\right)}}{3(-\mathrm{i}(\tau-\bar{\tau}))\left(-\mathrm{i}(T-\bar{T})+k\left(H^{a}, \bar{H}^{a}\right) f\left(U^{A}, \bar{U}^{A}\right)^{-\frac{1}{3}}\right)^{2} f\left(U^{A}, \bar{U}^{A}\right)^{\frac{1}{3}}}, \\
c=\frac{e^{-8 \mathcal{A}\left(H^{a}, \bar{H}^{a}\right)}}{6 M^{2}(-\mathrm{i}(\tau-\bar{\tau}))^{\frac{3}{2}}\left(-\mathrm{i}(T-\bar{T})+k\left(H^{a}, \bar{H}^{a}\right) f\left(U^{A}, \bar{U}^{A}\right)^{-\frac{1}{3}}\right)^{\frac{3}{2}} f\left(U^{A}, \bar{U}^{A}\right)^{\frac{5}{6}}} .
\end{gathered}
$$

${ }^{12}$ In the language of flat superspace, Eqs. (4.16) and (4.19) are defined as the chiral superfields

$$
\hat{f}_{\overline{D 3}}=\bar{D}^{2}\left(\frac{\bar{X} \bar{f}}{\bar{D}^{2} \bar{X}}\right), \quad \hat{M}_{i j}=\bar{D}^{2}\left(\frac{\bar{X} e^{-4 \mathcal{A}-2 u} m_{i j}}{\bar{D}^{2} \bar{X}(-\mathrm{i}(\tau-\bar{\tau}))^{\frac{1}{2}} f\left(U^{A}, \bar{U}^{A}\right)^{-\frac{1}{6}}}\right),
$$

and one has to require $\left\langle D^{2} X\right\rangle \neq 0$. 
In this case we then choose the superpotential to be

$$
W_{1}=W_{\mathrm{GVW}}+W_{n p}+M^{2} X .
$$

As an alternative, one can take a Kähler potential of the type

$$
K_{2}=-\log (-\mathrm{i}(\tau-\bar{\tau}))-3 \log \left[(-\mathrm{i}(T-\bar{T})) f\left(U^{A}, \bar{U}^{A}\right)^{\frac{1}{3}}+k\left(H^{a}, \bar{H}^{a}\right)\right]-3 \log \left(1-a X \bar{X}-b \delta_{i \bar{\jmath}} Y^{i} \bar{Y}^{\bar{\jmath}}\right)
$$

and give a mass to the fermions using the superpotential

$$
W_{2}=W_{\mathrm{GVW}}+W_{n p}+M^{2} X+\frac{1}{2} \hat{M}_{i j} Y^{i} Y^{j}
$$

where

$$
\hat{M}_{i j}=\Sigma\left(\frac{\left(\bar{X}^{0} e^{-\frac{K}{6}} \bar{X}\right) e^{-4 \mathcal{A}-2 u} m_{i j}}{\Sigma\left(\bar{X}^{0} e^{-\frac{K}{6}} \bar{X}\right)(-\mathrm{i}(\tau-\bar{\tau}))^{\frac{1}{2}} f\left(U^{A}, \bar{U}^{A}\right)^{-\frac{1}{6}}}\right) .
$$

In both cases $M^{2}=\sqrt{2}$ and $m_{i j}$ is given in Eq. (2.32). The supergravity multiplets $X, Y^{i}, P_{L} \Lambda_{\alpha}$, and $H^{a}$ describing the world-volume fields are constrained as

$$
X^{2}=0, \quad X Y^{i}=0, \quad X P_{L} \Lambda_{\alpha}=0, \quad X \bar{H}^{a}=\text { chiral }
$$

and contain, respectively, the Goldstino, the triplet of massive fermions, the $\mathrm{U}(1)$ gauge vector, and the three complex scalars as independent physical d.o.f.

Given a Kähler potential and a superpotential, one can use the standard rules of $\mathcal{N}=1$ supergravity to calculate the scalar potential $V^{\text {SUGRA }}=e^{K}\left(|D W|^{2}-3|W|^{2}\right)$. This will contain an uplift term which exactly matches the antiD3-brane contribution (2.12),

$$
V^{\text {SUGRA }} \supset V_{\overline{D 3}}(H, \bar{H})=2 e^{4 \mathcal{A}(H, \bar{H})-8 u} .
$$

\section{A. On the modular invariance of the Goldstino and matter sector}

As a consistency check for our result, we would like to analyze the behavior of the action (5.1) under modular transformations. Since the analysis of the $X, Y^{i}$ sector is quite different from that of the field strength multiplet $P_{L} \Lambda_{\alpha}$, we start from the former and we discuss the latter separately afterwards.

Therefore, neglecting for the moment the couplings involving the vector field, the original anti-D3-brane action (2.35) has to be invariant under $\operatorname{SL}(2, \mathbb{R})$ transformations,

$$
\tau \rightarrow \frac{a \tau+b}{c \tau+d}, \quad G_{3} \rightarrow \frac{G_{3}}{c \tau+d} .
$$

These imply that the world-volume fermions have to transform as

$$
P_{L} \lambda \rightarrow e^{-\mathrm{i} \delta} P_{L} \lambda, \quad P_{L} \chi^{i} \rightarrow e^{-\mathrm{i} \delta} P_{L} \chi^{i}
$$

where we defined the phase [27]

$$
e^{-2 \mathrm{i} \delta}=\left(\frac{c \bar{\tau}+d}{c \tau+d}\right)^{\frac{1}{2}}
$$

In particular, the transformation of the triplet of fermions $P_{L} \chi^{i}$ can be deduced by looking, e.g., at their mass term in Eq. (2.35). Indeed, Eq. (5.14) together with

$$
m_{i j} \rightarrow e^{2 i \delta} m_{i j}
$$

[which follows from Eq. (5.13)] imply that $m_{i j} \bar{\chi}^{i} P_{L} \chi^{j}$ is modular invariant. Once the transformation of $P_{L} \chi^{i}$ is fixed, it seems natural to let $P_{L} \lambda$ transform in the same way, since these four fermions originally resided in the same $\mathcal{N}=4$ multiplet. However, as we stressed previously, it is important to keep in mind that the Goldstino component field is not a physical d.o.f. in supergravity. Therefore, we will not insist on studying its modular properties further at the component level; instead, we will gain information by analyzing the couplings of the multiplet $X$, which (beside the Goldstino) contains the auxiliary field $F$.

Since $G_{3}$ is transforming under modular transformations, for consistency of the superpotential of the supergravity theory we have to require that the Goldstino multiplet transforms as well, namely, 


$$
X \rightarrow \frac{X}{c \tau+d}
$$

Such a requirement, in turn, implies that $\frac{X \bar{X}}{\tau-\bar{\tau}}$ is modular invariant, since $\operatorname{Im}(\tau) \rightarrow \frac{\operatorname{Im}(\tau)}{|c \tau+d|^{2}}$, and it fixes the coupling between the axiodilaton and the nilpotent multiplet $X$ in the Kähler potential according to the following reasoning. For a variation of the superpotential of the type

$$
W \rightarrow \frac{W}{c \tau+d},
$$

the Kähler potential is allowed to vary as

$$
K \rightarrow K+\log |c \tau+d|^{2}
$$

so that, if the supergravity theory is Kähler invariant, then it is also modular invariant. From the form of the Kähler potential (5.3) we can see that all of the freedom of $K$ to transform under modular transformations is already exhausted by the first term, namely, $-\log (-\mathrm{i}(\tau-\bar{\tau}))$, and thus all of the remaining couplings have to be modular invariant. With a similar argument, we can conclude that $\delta_{i \bar{\jmath}} \frac{Y^{i} \bar{Y} \bar{\jmath}}{\tau-\bar{\tau}}$ is modular invariant if $Y^{i}$ transforms as $X$, namely,

$$
Y^{i} \rightarrow \frac{Y^{i}}{c \tau+d}
$$

By direct inspection one can finally check that all of the remaining couplings in the Kähler potential and the superpotential have the correct behavior under modular transformations, for both choices of $K$ and $W$ that we presented before. In particular, in the case in which the multiplet $X$ is nilpotent, it is immediately seen that $\frac{X}{\Sigma\left(X^{0} X\right)}$ is modular invariant,

$$
\frac{X}{\bar{\Sigma}\left(X^{0} X\right)} \rightarrow \frac{X /(c \tau+d)}{\bar{\Sigma}\left(X^{0} X /(c \tau+d)\right)}=\frac{X}{\bar{\Sigma}\left(X^{0} X\right)},
$$

where we used the fact that the terms in which the operator $\bar{\Sigma}$ acts on $\tau$ are vanishing due to the constraint $X^{2}=0$. This observation can be helpful in proving the modular invariance of the proposed supergravity action, in particular for what concerns the superpotential mass term for the fermions.

\section{B. Self-duality of the vector}

As explained in the seminal paper [91], when a vector field is coupled to the axiodilaton, the original $\mathrm{U}(1)$ duality group is enhanced to $\operatorname{SL}(2, \mathbb{R})$. This corresponds precisely to the group of modular transformations we have discussed so far. With respect to the previous discussion, however, under duality rotations the action of the vector multiplet is not expected to be invariant. Indeed, the authors of
Ref. [91] calculated the general form of the induced variation. A duality transformation is in fact a symmetry of the equations of motion of the vector field, which is sometimes called self-duality since it exchanges the electric field strength with its magnetic dual and the gauge coupling with its inverse. In the following we check that the vector multiplet part of the anti-D3-brane action enjoys this property, namely, that it is on-shell equivalent to an action of the same functional form, in which the vector field is exchanged with its dual and the gauge kinetic function with its inverse:

$$
P_{L} \Lambda_{\alpha} \leftrightarrow P_{L} \Lambda_{D \alpha}, \quad \hat{f}_{\overline{D 3}} \leftrightarrow\left(\hat{f}_{\overline{D 3}}\right)^{-1} .
$$

Since self-duality is an on-shell property, we are allowed to use any form of the action which reduces on shell to the vector multiplet part of the anti-D3-brane action,

$$
S_{V}=-\frac{1}{4}\left[\hat{f}_{\overline{D 3}} \bar{\Lambda} P_{L} \Lambda\right]_{F} .
$$

In particular, it is convenient to relax the constraint (3.19) on the vector multiplet and impose it by means of a Lagrange multiplier. Therefore, we consider the action

$\tilde{S}_{V}=-\frac{1}{4}\left[\hat{f}_{\overline{D 3}} \bar{\Lambda} P_{L} \Lambda\right]_{F}+\frac{1}{2}\left[\bar{\Phi} P_{L} \Lambda X\right]_{F}+\left[\frac{i}{2} \bar{\Lambda}_{D} P_{L} \Lambda\right]_{F}$,

where $P_{L} \Lambda_{\alpha}$ is chiral but otherwise unconstrained, $P_{L} \Phi_{\alpha}$ is a Lagrange multiplier chiral multiplet that implements the constraint (3.19), and $P_{L} \Lambda_{D \alpha}$ is the dual of $P_{L} \Lambda_{\alpha}$. In particular, the chiral multiplet $P_{L} \Lambda_{\alpha}$ does not satisfy any Bianchi identity. It is also convenient to express the dual multiplet $P_{L} \Lambda_{D \alpha}$ as

$$
P_{L} \Lambda_{D \alpha}=\Sigma\left(\mathcal{D}_{\alpha} U\right)
$$

where $\mathcal{D}_{\alpha}$ is an operator, analogous to the superspace derivative [92], that maps

$$
\mathcal{D}_{\alpha}:(w, c) \rightarrow\left(w+\frac{1}{2}, c-\frac{3}{2}\right)
$$

and $U$ is a vector multiplet with vanishing weights. Notice that, by using the properties of the $\Sigma$ and $\mathcal{D}_{\alpha}$ operators, up to boundary terms we have

$$
\begin{aligned}
{\left[i \bar{\Lambda}_{D} P_{L} \Lambda\right]_{F} } & =\left[\mathrm{i} \Sigma\left(\mathcal{D}^{\alpha} U P_{L} \Lambda_{\alpha}\right)\right]_{F} \\
& =\left[\mathrm{i} \mathcal{D}^{\alpha} U P_{L} \Lambda_{\alpha}-\mathrm{i} \overline{\mathcal{D}}^{\alpha} U P_{R} \Lambda_{\alpha}\right]_{D} \\
& =-\left[\mathrm{i} U \mathcal{D}^{\alpha} P_{L} \Lambda_{\alpha}-\mathrm{i} U \overline{\mathcal{D}}^{\alpha} P_{R} \Lambda_{\alpha}\right]_{D} .
\end{aligned}
$$

As a consistency check, we first verify that by integrating out $U$ we get back the original action (5.23). The variation of $U$ gives 


$$
\delta U: \mathcal{D}^{\alpha} P_{L} \Lambda_{\alpha}=\overline{\mathcal{D}}^{\alpha} P_{R} \Lambda_{\alpha}
$$

which is the supersymmetric form of the Bianchi identities, implying that $P_{L} \Lambda_{\alpha}$ is the field strength of a vector multiplet $V: P_{L} \Lambda_{\alpha}=\Sigma\left(\mathcal{D}_{\alpha} V\right)$. Inserting this result into Eq. (5.24), the action correctly reduces to Eq. (5.23). On the other hand, by integrating out the Lagrange multiplier $P_{L} \Phi_{\alpha}$ and the unconstrained chiral multiplet $P_{L} \Lambda_{\alpha}$, we obtain

$$
\begin{gathered}
\delta P_{L} \Phi_{\alpha}: P_{L} \Lambda_{\alpha} X=0, \\
\delta P_{L} \Lambda_{\alpha}: \hat{f}_{\bar{D} 3} P_{L} \Lambda_{\alpha}=\mathrm{i} P_{L} \Lambda_{D \alpha}-P_{L} \Phi_{\alpha} X .
\end{gathered}
$$

By multiplying the second equation by $X$ and using the assumption that $X$ is nilpotent, we obtain the additional onshell information

$$
P_{L} \Lambda_{D \alpha} X=0
$$

which means that the fermion in the dual multiplet $P_{L} \Lambda_{D \alpha}$ is removed. Inserting Eq. (5.30) back into the action (5.24) and also using Eq. (5.31), we eventually obtain the on-shell expression

$$
\tilde{S}_{V}=-\frac{1}{4}\left[\hat{f}_{\bar{D} 3}^{-1} \bar{\Lambda}_{D} P_{L} \Lambda_{D}\right]_{F}
$$

Thus, we have shown that the vector multiplet sector of the anti-D3-brane action enjoys self-duality. Finally, we notice that, due to the nilpotent constraint on $X, \hat{f}_{\overline{D 3}}$ satisfies the property

$$
\left(\hat{f}_{\overline{D 3}}(\bar{f})\right)^{-1}=\hat{f}_{\overline{D 3}}\left(\bar{f}^{-1}\right),
$$

since

$$
\begin{aligned}
\left(\hat{f}_{\overline{D 3}}(\bar{f})\right)^{-1} & =\Sigma\left(\frac{\bar{X}^{0} e^{-\frac{K}{6}} \bar{X}}{\Sigma\left(\bar{X}^{0} e^{-\frac{K}{6}} \bar{X} \bar{f}\right)}\right) \\
& =\Sigma\left(\frac{\bar{X}^{0} e^{-\frac{K}{6}} \bar{X}}{\Sigma\left(\bar{X}^{0} e^{-\frac{K}{6}} \bar{X}\right) \bar{f}}\right)=\hat{f}_{\overline{D 3}}\left(\bar{f}^{-1}\right) .
\end{aligned}
$$

As a consequence, for the particular choice $f(\tau)=-\mathrm{i} \tau$, the transformation $\hat{f}_{\overline{D 3}} \rightarrow\left(\hat{f}_{\overline{D 3}}\right)^{-1}$ corresponds to $\tau \rightarrow-1 / \tau$. This, together with $\tau \rightarrow \tau+1$ (which is a trivial symmetry of the action), generates the full $\operatorname{SL}(2, \mathbb{R})$ group.

\section{CONCLUSION}

In this paper we studied the low-energy effective action for the KKLT scenario [1]. In particular, we kept track of all world-volume fields on the anti-D3-brane, i.e., the vector field, the scalars, and the fermions. Following Ref. [46], we showed how the corresponding world-volume fields couple to closed-string moduli, i.e., the axiodilaton, the complex structure moduli, and the single Kähler modulus. We then rewrote this $4 \mathrm{D}$ effective action in a manifestly supersymmetric way, making use of constrained multiplets [74] that have played an important role in recent advances in our understanding of supergravity (see, e.g., Refs. [77,93-97]).

Our manifestly supersymmetric $4 \mathrm{D} \mathcal{N}=1$ action shows that the anti-D3-brane in the KKLT scenario breaks supersymmetry spontaneously. It also provides a useful reformulation of the anti-D3-brane action that should facilitate future, more phenomenological studies of antibranes in string compactifications. Furthermore, it goes beyond what was in the literature in an important way. Indeed, initial studies of the uplift term in the KKLT scenario in supergravity have focused on the Goldstino, which can be packaged into a nilpotent chiral multiplet $[7,8]$, but the bosonic world-volume fields on the anti-D3brane have been neglected in this analysis because they can be projected out by placing the anti-D3-brane on top of an O3-plane [9,10,98]. This work culminated in Ref. [27], where the full action for all world-volume fermionsincluding their couplings to the closed-string moduli-was derived.

When adding the bosonic world-volume fields, we followed the proposal of Refs. [21,22] of how one can package the bosons into constrained $\mathcal{N}=1$ multiplets. While this choice might not be unique, it allowed us to rewrite the action in a manifestly supersymmetric way. One particular challenge one faces is related to the $U(1)$ gauge field on the anti-D3-brane. Recall that the gauge kinetic function $f$ for a D3-brane in the KKLT background is given by the axion dilaton $f(\tau)=-\mathrm{i} \tau=-\mathrm{i}\left(C_{0}+\mathrm{i} e^{-\phi}\right)$. This is and has to be a holomorphic function. However, the antiD3-brane has the opposite sign in the Wess-Zumino part of its action and therefore the $\mathrm{U}(1)$ world-volume gauge field naturally couples to $\mathrm{i} \bar{\tau}=-\mathrm{i}\left(-C_{0}+\mathrm{i} e^{-\phi}\right)$. The latter is antiholomorphic and such a gauge kinetic function is forbidden by supersymmetry. As explained in Sec. IV, we resolved this puzzle by using a construction inspired by the recently discovered new $D$-term in supergravity [28].

While our paper completes the study of a single anti-D3brane in the KKLT setup, there are a variety of future research directions that should be pursued:

(1) It should be straightforward to generalize our results to a stack of $N_{\overline{\mathrm{D} 3}}$ anti-D3-branes by simply promoting the world-volume fields to fields that transform in the adjoint of $\mathrm{SU}\left(N_{\overline{\mathrm{D}} 3}\right)$. This should amount to inserting traces into our formulas and adding commutator terms that appear in general [46] but vanish in the Abelian case. Note, however, that such a stack of anti-D3-branes in the KKLT background would want to polarize into an NS5-brane [5]. Two recent papers [25,31] studied the effective NS5-brane theory that arises from a stack of anti-D3-branes in the Klebanov-Strassler throat geometry [62]. 
The authors showed that in the metastable minimum supersymmetry is nonlinearly realized. This nonlinearly realized and spontaneously broken supersymmetry can be linearly realized if one includes a tower of massive Kaluza-Klein states [31]. It would be interesting to study the polarization process in more detail and include all world-volume fields on the anti-D3-branes.

(2) Since a reformulation in terms of constrained multiplets is not necessarily unique, one should explore other supersymmetric formulations of the action. For example, in Refs. [23,27] the authors also discussed a constrained vector superfield that contains the Goldstino as the only d.o.f. This vector superfield can replace the nilpotent chiral superfield and generates the KKLT uplift term via a $D$-term. It is conceivable that for the other constrained multiplets there are also alternative formulations that should be explored.

(3) Our results should be extendible to the large-volume scenario $[99,100]$. There, the AdS vacuum breaks supersymmetry already before adding the uplifting anti-D3-brane. This means that the Goldstino is always a combination of closed-string fermions and the fermions on the anti-D3-brane. Nevertheless, one should be able to describe the anti-D3-brane action in the same way that we did here. In particular, the nilpotent chiral superfield still consists of only a world-volume fermion.

(4) Recently it was shown that the anti-D3-brane in the KKLT scenario is one particular case of flux compactifications with anti-D $p$-branes that can all be described by a $4 \mathrm{D}$ effective $\mathcal{N}=1$ supergravity action that includes a nilpotent chiral multiplet [101]. It would be interesting to work out the proper description of the world-volume fields for anti-D $p$ branes with $p>3$. While this is not easy, one should be able to adapt existing results for the light d.o.f. on supersymmetric branes (see, e.g., Refs. [102-106]) since the DBI action for branes and antibranes is the same.

(5) It would be very interesting to derive the brane action in a nontrivial background beyond quadratic order in the fermions. In particular, the quartic terms play an important role in the study of the tendimensional lift of the 4D KKLT solution [50-61]. While technically challenging to obtain, these higherorder fermionic terms would have applications well beyond the study of anti-D-branes in flux compactifications.

We hope to study some of these issues in the future.

\section{ACKNOWLEDGMENTS}

We are grateful to Fotis Farakos, Renata Kallosh, Liam McAllister, Susha Parameswaran, Harald Skarke, Flavio
Tonioni, Magnus Tournoy, and Antoine van Proeyen for enlightening discussions and collaboration on related projects, and to Liam McAllister for comments on an earlier version of this draft. The work of N. C., C. R., and T. W. is supported by the FWF Grant No. P 30265. The work of Y. Y. was supported by the Stanford Institute of Theoretical Physics (SITP), the US National Science Foundation Grant No. PHY-1720397, and the Grant-inAid for JSPS Fellows (19J00494).

\section{APPENDIX A: SUPERSPACE}

In this Appendix we translate the relevant formulas into the language of superspace, following the conventions of Ref. [70]. In this case fermions are described by twocomponent Weyl spinors. We recall that a four-component spinor can be written in terms of a two-component one as $\Omega=\left(\begin{array}{l}\psi \\ \bar{\psi}\end{array}\right)$.

\section{Constrained superfields}

Here we describe constrained superfields in flat superspace.

(1) The nilpotent chiral Goldstino superfield is given by

$$
X=\frac{G^{2}}{2 F}+\sqrt{2} \theta G+\theta^{2} F \Leftrightarrow X^{2}=0,
$$

where $G_{\alpha}$ is the spin- $1 / 2$ Goldstino. Upon substituting $\theta \rightarrow \Theta$, this expression is also valid in supergravity.

(2) A chiral superfield $Y$, such that $X Y=0$, is instead

$$
Y=\frac{G \chi}{F}-\frac{G^{2}}{2 F^{2}} F^{Y}+\sqrt{2} \theta \chi+\theta^{2} F^{Y} .
$$

Upon substituting $\theta \rightarrow \Theta$, this expression is also valid in supergravity.

(3) A chiral superfield $H$

$$
H=h+\sqrt{2} \theta \chi^{H}+\theta^{2} F^{H}
$$

containing only the complex scalar in the lowest component is subjected to the constraint

$$
X \bar{D}_{\dot{\alpha}} \bar{H}=0,
$$

where $X^{2}=0$ is assumed. Its fermion and auxiliary fields are given by

$$
\chi^{H}=i \sigma^{\mu}\left(\frac{\bar{G}}{\bar{F}}\right) \partial_{\mu} h
$$

$$
F^{H}=-\partial_{\mu}\left(\frac{\bar{G}}{\bar{F}}\right) \bar{\sigma}^{\nu} \sigma^{\mu} \frac{\bar{G}}{\bar{F}} \partial_{\nu} h+\frac{\bar{G}^{2}}{2 \bar{F}^{2}} \square h .
$$


The generalization to supergravity can be found in Ref. [93].

(4) Given a real Abelian vector superfield $V$, the field strength chiral superfield is

$$
W_{\alpha}=-\frac{1}{4} \bar{D}^{2} D_{\alpha} V=-\mathrm{i} \Lambda_{\alpha}+L_{\alpha}^{\beta} \theta_{\beta}+\sigma_{\alpha \dot{\alpha}}^{\mu} \partial_{\mu} \bar{\Lambda}^{\dot{\alpha}} \theta^{2}
$$

where

$$
L_{\alpha}^{\beta}=\delta_{\alpha}^{\beta} D-\frac{\mathrm{i}}{2}\left(\sigma^{\mu} \sigma^{\nu}\right)_{\alpha}^{\beta} F_{\mu \nu} .
$$

The gaugino $\Lambda_{\alpha}$ can be removed via the constraint

$$
X W_{\alpha}=0
$$

which leads to

$$
\begin{aligned}
\Lambda_{\alpha}= & \frac{\mathrm{i}}{\sqrt{2} F} L_{\alpha}^{\beta} G_{\beta}-\frac{G^{2}}{2 F^{2}} \partial_{\mu}\left(\frac{\bar{G}_{\dot{\alpha}} \bar{L}_{\dot{\beta}}^{\dot{\alpha}}}{\sqrt{2} \bar{F}}\right) \bar{\sigma}^{\mu \dot{\beta} \gamma} \epsilon_{\gamma \alpha} \\
& -\mathrm{i} \frac{G^{2}}{2 F^{2}} \sigma_{\alpha \dot{\beta}}^{\mu} \bar{\sigma}^{\nu \dot{\beta} \gamma} \partial_{\mu}\left(\frac{\bar{G}^{2}}{2 \bar{F}^{2}} \partial_{\nu}\left(\frac{L_{\gamma}^{\delta} G_{\delta}}{\sqrt{2} F}\right)\right) \\
& -\frac{1}{2} \frac{G^{2}}{F^{2}} \frac{\bar{G}^{2}}{\bar{F}^{2}}\left(\partial\left(\frac{G}{\sqrt{2} F}\right)\right)^{2} \partial_{\mu}\left(\frac{\bar{G}_{\dot{\alpha}} \bar{L}_{\dot{\beta}}^{\dot{\alpha}}}{\sqrt{2} \bar{F}}\right) \bar{\sigma}^{\mu \dot{\beta} \gamma} \epsilon_{\gamma \alpha} .
\end{aligned}
$$

The supergravity expression for the removed gaugino can be found in Ref. [93].

\section{The anti-D3-brane supergravity Lagrangian}

The supergravity Lagrangian for the anti-D3-brane can be expressed in superspace as

$$
\mathcal{L}=-3 \int d^{4} \theta E e^{-\frac{K}{3}}+\frac{1}{4}\left(\int d^{2} \Theta 2 \mathcal{E} \hat{f}_{\overline{D 3}} \mathcal{W}^{\alpha} \mathcal{W}_{\alpha}+\text { c.c. }\right)+\left(\int d^{2} \Theta 2 \mathcal{E} W+\text { c.c. }\right)
$$

where $K$ is the Kähler potential, $W$ is the superpotential, and $\mathcal{W}_{\alpha}=-\frac{1}{4}\left(\overline{\mathcal{D}}^{2}-8 \mathcal{R}\right) \mathcal{D}_{\alpha} V$ is the field strength superfield. The anti-D3-brane gauge kinetic function is

$$
\hat{f}_{\overline{D 3}}=\left(\overline{\mathcal{D}}^{2}-8 \mathcal{R}\right)\left(\frac{\bar{X} \bar{f}}{\overline{\mathcal{D}}^{2} \bar{X}}\right)
$$

where $f(\tau)=-\mathrm{i} \tau$. Equations (5.3), (5.7) and (5.8), (5.9) we gave for $K$ and $W$ can be used directly without any modification. When using the second alternative, the superpotential mass for the fermions in $Y^{i}$ is generated by the chiral superfield

$$
\hat{M}_{i j}=\left(\overline{\mathcal{D}}^{2}-8 \mathcal{R}\right)\left(\frac{e^{-4 \mathcal{A}-2 u} \bar{X} m_{i j}}{\overline{\mathcal{D}}^{2} \bar{X}(-\mathrm{i}(\tau-\bar{\tau}))^{\frac{1}{2}} f\left(U^{A}, \bar{U}^{A}\right)^{-\frac{1}{6}}}\right),
$$

where $m_{i j}$ is given in Eq. (2.32).

We notice finally that Eqs. (A12) and (A13) can be written in an alternative form that may be preferred in some applications. Given a nilpotent Goldstino superfield $X$, we can define a superfield [23,107]

$$
\Gamma_{\alpha}=-2 \sqrt{2} \frac{\mathcal{D}_{\alpha} X}{\mathcal{D}^{2} X}
$$

It is possible to check that such a superfield satisfies

$$
\begin{aligned}
& \mathcal{D}_{\beta} \Gamma_{\alpha}=\sqrt{2} \epsilon_{\alpha \beta}\left(1-\overline{\mathcal{R}} \Gamma^{2}\right), \\
& \overline{\mathcal{D}}^{\dot{\beta}} \Gamma^{\alpha}=\sqrt{2} \mathrm{i}\left(\bar{\sigma}^{b} \Gamma\right)^{\dot{\beta}} \mathcal{D}_{b} \Gamma^{\alpha}+\frac{1}{2 \sqrt{2}} \Gamma^{2} B^{\dot{\beta} \alpha},
\end{aligned}
$$

where $\mathcal{D}_{a} \Gamma_{\alpha}=e_{b}^{m} \mathcal{D}_{m} \Gamma_{\alpha}-\frac{1}{2} \psi_{a}^{\beta} \mathcal{D}_{\beta} \Gamma_{\alpha}-\frac{1}{2} \bar{\psi}_{b \dot{\beta}} \overline{\mathcal{D}}^{\dot{\beta}} \Gamma_{\alpha}$ is the supercovariant derivative in superspace and the definition of the superfield $B_{\alpha \dot{\beta}}$ can be found in Ref. [70]. These are the conditions given in Ref. [108] in order for $\Gamma_{\alpha}$ to describe a Goldstino. Indeed, the lowest component $\Gamma_{\alpha} \mid=\gamma_{\alpha}$, is the (chiral) Volkov-Akulov Goldstino, while all of the other fields inside $\Gamma_{\alpha}$ are removed. With this new ingredient at our disposal, the gauge kinetic function and the superpotential mass can be expressed in a more compact form as

$$
\begin{aligned}
& \hat{f}_{\overline{D 3}}=-\frac{1}{8}\left(\overline{\mathcal{D}}^{2}-8 \mathcal{R}\right)\left(\bar{\Gamma}^{2} \bar{f}\right), \\
& \hat{M}_{i j}=-\frac{1}{8}\left(\overline{\mathcal{D}}^{2}-8 \mathcal{R}\right)\left(\bar{\Gamma}^{2} \frac{e^{-4 \mathcal{A}-2 u}}{(-\mathrm{i}(\tau-\bar{\tau}))^{\frac{1}{2}} f\left(U^{A}, \bar{U}^{A}\right)^{-\frac{1}{6}}} m_{i j}\right) .
\end{aligned}
$$

Their lowest components in the unitary gauge $\gamma_{\alpha}=0$ are

$$
\hat{f}_{\overline{D 3}}\left|=\bar{f}, \quad \hat{M}_{i j}\right|=\frac{e^{-4 \mathcal{A}-2 u}}{(-\mathrm{i}(\tau-\bar{\tau}))^{\frac{1}{2}} f\left(U^{A}, \bar{U}^{A}\right)^{-\frac{1}{6}}} m_{i j} .
$$

We stress that such a construction is general, namely, given an arbitrary superfield $\Phi$, which can also be composite, the chiral superfield 


$$
\hat{\Phi}=-\frac{1}{8}\left(\overline{\mathcal{D}}^{2}-8 \mathcal{R}\right)\left(\bar{\Gamma}^{2} \Phi\right)
$$

has the lowest component

$$
\hat{\Phi} \mid=\Phi+\text { fermions }
$$

and the fermionic terms vanish in the unitary gauge. It satisfies the constraint

$$
\Gamma^{2} \bar{\Gamma}^{2} \hat{\Phi}=\Gamma^{2} \bar{\Gamma}^{2} \Phi
$$

For completeness, we give the components of $\hat{f}_{\overline{D 3}}$ in global supersymmetry. The chiral superfield $\hat{f}_{\overline{D 3}}$ can be expanded in superspace as

$$
\hat{f}_{\overline{D 3}}=\hat{f}_{\overline{D 3}}\left|+\theta^{\alpha} D_{\alpha} \hat{f}_{\overline{D 3}}\right|-\frac{1}{4} \theta^{2} D^{2} \hat{f}_{\overline{D 3}} \mid,
$$

where

$$
\hat{f}_{\overline{D 3}} \mid=\bar{f}+\frac{2 \bar{D}_{\dot{\alpha}} \bar{X} \bar{D}^{\dot{\alpha}} \bar{f}+\bar{X} \bar{D}^{2} \bar{f}}{\bar{D}^{2} \bar{X}}
$$

$$
\begin{aligned}
D_{\alpha} \hat{f}_{\overline{D 3}} \mid=-4 \mathrm{i} \partial_{\alpha \dot{\alpha}} \bar{X} & \frac{\bar{D}^{\dot{\alpha}} \bar{f}}{\bar{D}^{2} \bar{X}}-4 \mathrm{i} \partial_{\alpha \dot{\alpha}} \bar{f} \frac{\bar{D}^{\dot{\alpha}} \bar{X}}{\bar{D}^{2} \bar{X}}-4 \mathrm{i} \frac{\bar{X}}{\bar{D}^{2} \bar{X}} \partial_{\alpha \dot{\alpha}} \bar{D}^{\dot{\beta}} \bar{f}+4 \mathrm{i} \partial_{\alpha \dot{\alpha}} \bar{D}^{\dot{\alpha}} \bar{X}\left(\frac{2 \bar{D}_{\dot{\beta}} \bar{X} \bar{D}^{\dot{\beta}} \bar{f}+\bar{X} \bar{D}^{2} \bar{f}}{\left(\bar{D}^{2} \bar{X}\right)^{2}}\right), \\
D^{2} \hat{f}_{\overline{D 3}} \mid= & -16 \frac{\partial_{\alpha \dot{\alpha}} \bar{X} \partial^{\dot{\alpha} \alpha} \bar{f}}{\bar{D}^{2} \bar{X}}-32 \frac{\partial_{\alpha \dot{\alpha}} \bar{X} \bar{D}^{\dot{\alpha}} \bar{f} \partial^{\dot{\beta} \alpha} \bar{D}_{\dot{\beta}} \bar{X}}{\left(\bar{D}^{2} \bar{X}\right)^{2}}-32 \frac{\partial_{\alpha \dot{\alpha}} \bar{f} \bar{D}^{\dot{\alpha}} \bar{X} \partial^{\dot{\beta} \alpha} \bar{D}_{\dot{\beta}} \bar{X}}{\left(\bar{D}^{2} \bar{X}\right)^{2}} \\
& +16 \frac{\bar{X} \square \bar{f}}{\bar{D}^{2} \bar{X}}-32 \frac{\bar{X} \partial^{\dot{\beta} \alpha} \bar{D}_{\dot{\beta}} \bar{X} \partial_{\alpha \dot{\gamma}} \bar{D}^{\dot{\gamma}} \bar{f}}{\left(\bar{D}^{2} \bar{X}\right)^{2}}-16 \frac{\square \bar{X}\left(2 \bar{D}_{\dot{\alpha}} \bar{X} \bar{D}^{\dot{\alpha}} \bar{f}+\bar{X} \bar{D}^{2} \bar{f}\right)}{\left(\bar{D}^{2} \bar{X}\right)^{2}} \\
& -64 \frac{\partial_{\alpha \dot{\beta}} \bar{D}^{\dot{\beta}} \bar{X} \bar{D}_{\dot{\gamma}} \bar{X} \bar{D}^{\dot{\gamma}} \bar{f} \partial^{\dot{\alpha} \alpha} \bar{D}_{\dot{\alpha}} \bar{X}}{\left(\bar{D}^{2} \bar{X}\right)^{3}}-32 \frac{\bar{X} \partial_{\alpha \dot{\beta}} \bar{D}^{\dot{\beta}} \bar{X} \bar{D}^{2} \bar{f} \partial^{\dot{\alpha} \alpha} \bar{D}_{\dot{\alpha}} \bar{X}}{\left(\bar{D}^{2} \bar{X}\right)^{3}} .
\end{aligned}
$$

$$
\begin{aligned}
S_{\text {FI new }} & =-\xi\left[\frac{\omega^{2} \bar{\omega}^{2}}{\sum\left(\bar{\omega}^{2}\right) \bar{\Sigma}\left(\omega^{2}\right)} X^{0} \bar{X}^{0} \mathrm{D}\right]_{D} \\
& =-\xi \int d^{4} x \sqrt{-g_{4}} X^{0} \bar{X}^{0} \mathrm{D}+\ldots,
\end{aligned}
$$

where the dots stand for fermionic terms. In this expression, $X^{0}$ is the compensator chiral multiplet, D is a real multiplet which has the auxiliary field in the lowest component [see Eq. (3.3) of Ref. [28]], and we defined the multiplets

$$
\omega^{2}=\frac{\bar{\Lambda} P_{L} \Lambda}{\left(X^{0} \bar{X}^{0}\right)^{2}}, \quad \bar{\omega}^{2}=\frac{\bar{\Lambda} P_{R} \Lambda}{\left(X^{0} \bar{X}^{0}\right)^{2}} .
$$

$\Sigma$ and $\bar{\Sigma}$ are the superconformal generalizations of the chiral projectors $\bar{D}^{2}$ and $D^{2}$ of superspace. They act on multiplets with weights (Weyl, chiral) $=(w, \pm(w-2))$ and produce, respectively, chiral and antichiral multiplets, namely,

$$
\begin{aligned}
& \Sigma:(w, w-2) \rightarrow(w+1, w+1), \\
& \bar{\Sigma}:(w,-w+2) \rightarrow(w+1,-w-1) .
\end{aligned}
$$

More details about these operators can be found, e.g., in Ref. [82], where they are denoted as $T$ and $\bar{T}$. We also need the components of the chiral multiplet 


$$
\begin{aligned}
\bar{\Lambda} P_{L} \Lambda= & \left\{\bar{\Lambda} P_{L} \Lambda, \sqrt{2} P_{L}\left(\mathrm{iD}-\frac{1}{2} \hat{F}\right) \Lambda, 2 \bar{\Lambda} P_{L} \not D \Lambda\right. \\
& \left.+\hat{F}^{-} \cdot \hat{F}^{-}-\mathrm{D}^{2}\right\},
\end{aligned}
$$

from which one can calculate

$$
\bar{\Sigma}\left(\omega^{2}\right)=\left(X^{0} \bar{X}^{0}\right)^{-2}\left(\frac{1}{2} F_{\mu \nu} F^{\mu \nu}+\frac{i}{4} \frac{\epsilon^{\mu \nu \rho \sigma}}{\sqrt{-g_{4}}} F_{\mu \nu} F_{\rho \sigma}-\mathrm{D}^{2}+\ldots\right),
$$

where the dots stand for fermionic terms. We recall that we are following the notation and the conventions of Ref. [69].

The important property of the coupling (B2) is that its pure bosonic sector contains just a term linear in the auxiliary field $D$, while all of the remaining fermionic terms are required by superconformal symmetry. After gauge fixing the superconformal symmetry to Poincaré supergravity, $X^{0}=\kappa^{-1} e^{\frac{K}{6}}$, the new $D$-term reduces to $(\kappa=1)$

$$
S_{\text {FI new }}=-\xi \int d^{4} x \sqrt{-g_{4}} e^{\frac{K}{3}} \mathrm{D}+\ldots,
$$

and therefore, when this is added to the standard supergravity action (4.8) for the vector multiplet, the auxiliary field $D$ acquires a nonvanishing vacuum expectation value and supersymmetry is spontaneously broken. Then, by setting the Goldstino to zero by a unitary gauge choice, all of the fermionic interactions in Eq. (B7) vanish. Notice that the presence of this coupling spoils the Kähler invariance of the theory, since the Kähler potential appears explicitly in the action. The Kähler invariant version of Eq. (B2) was constructed in Ref. [87] and it amounts to replacing $X^{0} \bar{X}^{0} \rightarrow X^{0} \bar{X}^{0} e^{-\frac{K}{3}}$ in order to cancel the undesired dependence on $K$ in the Poincaré frame. In what follows we systematically perform this replacement.

The logic behind Eq. (B2) is the following. First, notice that it is constructed out of the two real multiplets

$$
R_{1}=\frac{\omega^{2} \bar{\omega}^{2}}{\Sigma\left(\bar{\omega}^{2}\right) \bar{\Sigma}\left(\omega^{2}\right)}, \quad R_{2}=X^{0} \bar{X}^{0} e^{-\frac{K}{3}} \mathrm{D},
$$

with weights $(-2,0)$ and $(4,0)$, respectively, and that the component expansion starts precisely with the lowest component of $R_{2}$. This means that the role of $R_{1}$ is to provide higher-order fermionic interactions which are needed in order to write down a consistent superconformal embedding of $R_{2}$. The procedure can be easily generalized in order to construct the superconformal completion of any arbitrary real multiplet $R_{2}$ with weights $(4,0)$. A similar logic was followed in Ref. [109] by using only chiral multiplets.
In Sec. IV B we are interested in deforming a given supergravity action in a supersymmetric way with a theta term

$$
S_{\theta \text {-term }}=-\frac{1}{4} \int d^{4} x \operatorname{Im}(f) \epsilon^{\mu \nu \rho \sigma} F_{\mu \nu} F_{\rho \sigma}+\ldots,
$$

where the dots are additional couplings which are needed from supersymmetry and which we would like to determine. The standard procedure to find them consists in varying Eq. (B9) and adding the required terms in order to cancel the total variation and obtain a superconformal invariant coupling. This strategy is conceptually simple but tedious and it is not clear at which step it is going to end. However, when supersymmetry is spontaneously broken the problem can be solved at once by considering the multiplet $R_{1}$ as defined in Eq. (B8) and multiplying it by

$$
R_{2}=\operatorname{Im}(f) \epsilon^{\mu \nu \rho \sigma} F_{\mu \nu} F_{\rho \sigma}
$$

which has to be thought of as a real multiplet with weights $(4,0)$. In particular, $f(\tau)=-\mathrm{i} \tau$ can be understood as the lowest component of a chiral multiplet with vanishing weight. This strategy is formally correct, but there is one additional subtlety one needs to be careful about in our case. In the anti-D3-brane action we propose, the auxiliary field of the vector multiplet does not acquire a vacuum expectation value, since supersymmetry is spontaneously broken by the auxiliary field $F$ of $X$. As a consequence, we are not allowed to divide by the quantity $\bar{\Sigma}\left(\omega^{2}\right)$, as it vanishes in the vacuum. However, in the case in which the field strength multiplet is constrained as in Eq. (3.19), we can use the identity (proven in Ref. [28])

$\frac{\omega^{2} \bar{\omega}^{2}}{\Sigma\left(\bar{\omega}^{2}\right) \bar{\Sigma}\left(\omega^{2}\right)}=\frac{X^{0} \bar{X}^{0} X \bar{X}}{\Sigma\left(\bar{X}^{0} \bar{X}\right) \bar{\Sigma}\left(X^{0} X\right)}=\frac{X^{0} \bar{X}^{0} e^{-\frac{K}{3}} X \bar{X}}{\Sigma\left(\bar{X}^{0} e^{-\frac{K}{6}} \bar{X}\right) \bar{\Sigma}\left(X^{0} e^{-\frac{K}{6}} X\right)}$

to formally trade $\omega^{2}$ for the nilpotent $X$ inside $R_{1}$. In particular, the right-hand side is well defined in our setup, since the denominator can never vanish. We are therefore in a position to propose the following superconformal invariant interaction:

$$
\begin{aligned}
S_{\theta \text {-term }} & =-\frac{1}{4}\left[\frac{X^{0} \bar{X}^{0} e^{-\frac{K}{3}} X \bar{X}}{\sum\left(\bar{X}^{0} e^{-\frac{K}{6}} \bar{X}\right) \bar{\Sigma}\left(X^{0} e^{-\frac{K}{6}} X\right)} \operatorname{Im}(f) \frac{\epsilon^{\mu \nu \rho \sigma}}{\sqrt{-g_{4}}} F_{\mu \nu} F_{\rho \sigma}\right]_{D} \\
& =-\frac{1}{4} \operatorname{Im}(f) \epsilon^{\mu \nu \rho \sigma} F_{\mu \nu} F_{\rho \sigma}+\ldots,
\end{aligned}
$$

which has the desired property of providing a consistent superconformal completion of Eq. (B9), much in the same way that Eq. (B2) provides it for the Fayet-Iliopoulos term (B1). We can also express the multiplet $\epsilon^{\mu \nu \rho \sigma} F_{\mu \nu} F_{\rho \sigma}$ in terms of the more familiar field strength multiplet $P_{L} \Lambda$. One can indeed check that 


$$
\left(X^{0} \bar{X}^{0} e^{-\frac{K}{3}}\right)^{2} \frac{1}{2 \mathrm{i}}\left[\bar{\Sigma}\left(\omega^{2}\right)-\Sigma\left(\bar{\omega}^{2}\right)\right]=\frac{1}{4} \frac{\epsilon^{\mu \nu \rho \sigma}}{\sqrt{-g_{4}}} F_{\mu \nu} F_{\rho \sigma}+\ldots
$$

where the dots stand for fermionic terms containing at least one gaugino $\Lambda_{\alpha}$ without derivatives acting on it. Due to the Grassmann nature of $\Lambda_{\alpha}$, these terms vanish if multiplied by $X \bar{X}$ and we directly have the constraint

$$
X \bar{X}\left(X^{0} \bar{X}^{0} e^{-\frac{K}{3}}\right)^{2} \frac{1}{2 \mathrm{i}}\left[\bar{\Sigma}\left(\omega^{2}\right)-\Sigma\left(\bar{\omega}^{2}\right)\right]=\frac{1}{4} X \bar{X} \frac{\epsilon^{\mu \nu \rho \sigma}}{\sqrt{-g_{4}}} F_{\mu \nu} F_{\rho \sigma},
$$

where every quantity can be understood as a full supersymmetric multiplet. The proposed coupling (B12) then becomes

$$
\begin{aligned}
S_{\theta \text {-term }} & =-\frac{1}{4}\left[\frac{X^{0} \bar{X}^{0} e^{-\frac{K}{3}} X \bar{X}}{\Sigma\left(\bar{X}^{0} e^{-\frac{K}{6}} \bar{X}\right) \bar{\Sigma}\left(X^{0} e^{-\frac{K}{6}} X\right)} \operatorname{Im}(f) \frac{\epsilon^{\mu \nu \rho \sigma}}{\sqrt{-g_{4}}} F_{\mu \nu} F_{\rho \sigma}\right]_{D} \\
& =-\frac{1}{2 \mathrm{i}}\left[\frac{X \bar{X}}{\sum\left(\bar{X}^{0} e^{-\frac{K}{6}} \bar{X}\right) \bar{\Sigma}\left(X^{0} e^{-\frac{K}{6}} X\right)}\left(X^{0} \bar{X}^{0} e^{-\frac{K}{3}}\right)^{3} \operatorname{Im}(f)\left(\bar{\Sigma}\left(\omega^{2}\right)-\Sigma\left(\bar{\omega}^{2}\right)\right)\right]_{D} \\
& =\left[\frac{X \bar{X}}{\Sigma\left(\bar{X}^{0} e^{-\frac{K}{6}} \bar{X}\right) \bar{\Sigma}\left(X^{0} e^{-\frac{K}{6}} X\right)}\left(X^{0} \bar{X}^{0} e^{-\frac{K}{3}}\right)^{3} \operatorname{Im}(f) \operatorname{Im}\left(\Sigma\left(\bar{\omega}^{2}\right)\right)\right]_{D} \\
& =-\frac{1}{4} \int d^{4} x \operatorname{Im}(f) \epsilon^{\mu \nu \rho \sigma} F_{\mu \nu} F_{\rho \sigma}+\ldots,
\end{aligned}
$$

which is the expression that appears in Eq. (4.9).

[1] S. Kachru, R. Kallosh, A. D. Linde, and S. P. Trivedi, De Sitter vacua in string theory, Phys. Rev. D 68, 046005 (2003).

[2] T. D. Brennan, F. Carta, and C. Vafa, The string landscape, the swampland, and the missing corner, Proc. Sci., TASI2017 (2017) 015 [arXiv:1711.00864].

[3] U. H. Danielsson and T. Van Riet, What if string theory has no de Sitter vacua?, Int. J. Mod. Phys. D 27, 1830007 (2018).

[4] E. Palti, The swampland: Introduction and review, Fortschr. Phys. 67, 1900037 (2019).

[5] S. Kachru, J. Pearson, and H. L. Verlinde, Brane / flux annihilation and the string dual of a nonsupersymmetric field theory, J. High Energy Phys. 06 (2002) 021.

[6] S. Ferrara, R. Kallosh, and A. Linde, Cosmology with nilpotent superfields, J. High Energy Phys. 10 (2014) 143.

[7] R. Kallosh and T. Wrase, Emergence of spontaneously broken supersymmetry on an anti-D3-brane in KKLT dS vacua, J. High Energy Phys. 12 (2014) 117.

[8] E. A. Bergshoeff, K. Dasgupta, R. Kallosh, A. Van Proeyen, and T. Wrase, $\overline{\mathrm{D} 3}$ and dS, J. High Energy Phys. 05 (2015) 058.

[9] R. Kallosh, F. Quevedo, and A. M. Uranga, String theory realizations of the nilpotent Goldstino, J. High Energy Phys. 12 (2015) 039.

[10] I. García-Etxebarria, F. Quevedo, and R. Valandro, Global string embeddings for the nilpotent Goldstino, J. High Energy Phys. 02 (2016) 148.
[11] S. Sugimoto, Anomaly cancellations in type I D-9-antiD-9 system and the USp(32) string theory, Prog. Theor. Phys. 102, 685 (1999).

[12] I. Antoniadis, E. Dudas, and A. Sagnotti, Brane supersymmetry breaking, Phys. Lett. B 464, 38 (1999).

[13] C. Angelantonj, Comments on open string orbifolds with a nonvanishing B(ab), Nucl. Phys. B566, 126 (2000).

[14] G. Aldazabal and A. M. Uranga, Tachyon free nonsupersymmetric type IIB orientifolds via Brane-anti-brane systems, J. High Energy Phys. 10 (1999) 024.

[15] C. Angelantonj, I. Antoniadis, G. D’Appollonio, E. Dudas, and A. Sagnotti, Type I vacua with brane supersymmetry breaking, Nucl. Phys. B572, 36 (2000).

[16] E. Dudas and J. Mourad, Consistent gravitino couplings in nonsupersymmetric strings, Phys. Lett. B 514, 173 (2001).

[17] G. Pradisi and F. Riccioni, Geometric couplings and brane supersymmetry breaking, Nucl. Phys. B615, 33 (2001).

[18] M. Bertolini, D. Musso, I. Papadimitriou, and H. Raj, A Goldstino at the bottom of the cascade, J. High Energy Phys. 11 (2015) 184.

[19] I. Bandos, L. Martucci, D. Sorokin, and M. Tonin, Brane induced supersymmetry breaking and de Sitter supergravity, J. High Energy Phys. 02 (2016) 080.

[20] K. Dasgupta, M. Emelin, and E. McDonough, Fermions on the antibrane: Higher order interactions and spontaneously broken supersymmetry, Phys. Rev. D 95, 026003 (2017). 
[21] B. Vercnocke and T. Wrase, Constrained superfields from an anti-D3-brane in KKLT, J. High Energy Phys. 08 (2016) 132.

[22] R. Kallosh, B. Vercnocke, and T. Wrase, String theory origin of constrained multiplets, J. High Energy Phys. 09 (2016) 063.

[23] I. Bandos, M. Heller, S. M. Kuzenko, L. Martucci, and D. Sorokin, The Goldstino brane, the constrained superfields and matter in $\mathcal{N}=1$ supergravity, J. High Energy Phys. 11 (2016) 109.

[24] S. Aoki and Y. Yamada, More on DBI action in 4D $\mathcal{N}=1$ supergravity, J. High Energy Phys. 01 (2017) 121.

[25] L. Aalsma, J. P. van der Schaar, and B. Vercnocke, Constrained superfields on metastable anti-D3-branes, J. High Energy Phys. 05 (2017) 089.

[26] R. Kallosh, A. Linde, D. Roest, and Y. Yamada, $\overline{D 3}$ induced geometric inflation, J. High Energy Phys. 07 (2017) 057.

[27] M. P. G. del Moral, S. Parameswaran, N. Quiroz, and I. Zavala, Anti-D3 branes and moduli in non-linear supergravity, J. High Energy Phys. 10 (2017) 185.

[28] N. Cribiori, F. Farakos, M. Tournoy, and A. van Proeyen, Fayet-Iliopoulos terms in supergravity without gauged Rsymmetry, J. High Energy Phys. 04 (2018) 032.

[29] N. Kitazawa, Brane SUSY breaking and the gravitino mass, J. High Energy Phys. 04 (2018) 081.

[30] C. Krishnan, H. Raj, and P. N. Bala Subramanian, On the KKLT Goldstino, J. High Energy Phys. 06 (2018) 092.

[31] L. Aalsma, M. Tournoy, J. P. Van Der Schaar, and B. Vercnocke, Supersymmetric embedding of antibrane polarization, Phys. Rev. D 98, 086019 (2018).

[32] N. Cribiori, F. Farakos, and M. Tournoy, Supersymmetric Born-Infeld actions and new Fayet-Iliopoulos terms, J. High Energy Phys. 03 (2019) 050.

[33] M. Grana, D3-brane action in a supergravity background: The Fermionic story, Phys. Rev. D 66, 045014 (2002).

[34] M. Grana, T. W. Grimm, H. Jockers, and J. Louis, Soft supersymmetry breaking in Calabi-Yau orientifolds with D-branes and fluxes, Nucl. Phys. B690, 21 (2004).

[35] D. Marolf, L. Martucci, and P. J. Silva, Fermions, T duality and effective actions for D-branes in bosonic backgrounds, J. High Energy Phys. 04 (2003) 051.

[36] P. K. Tripathy and S. P. Trivedi, D3 brane action and fermion zero modes in presence of background flux, J. High Energy Phys. 06 (2005) 066.

[37] L. Martucci, J. Rosseel, D. Van den Bleeken, and A. Van Proeyen, Dirac actions for D-branes on backgrounds with fluxes, Classical Quantum Gravity 22, 2745 (2005).

[38] E. Bergshoeff, F. Coomans, R. Kallosh, C. S. Shahbazi, and A. Van Proeyen, Dirac-Born-Infeld-Volkov-Akulov and deformation of supersymmetry, J. High Energy Phys. 08 (2013) 100.

[39] D. V. Volkov and V. P. Akulov, Is the neutrino a Goldstone particle?, Phys. Lett. 46B, 109 (1973).

[40] S. B. Giddings, S. Kachru, and J. Polchinski, Hierarchies from fluxes in string compactifications, Phys. Rev. D 66, 106006 (2002).
[41] J. Polchinski, String Theory. Vol. 2: Superstring Theory and Beyond (Cambridge University Press, Cambridge, England, 2007).

[42] A. R. Frey, G. Torroba, B. Underwood, and M. R. Douglas, The universal Kahler modulus in warped compactifications, J. High Energy Phys. 01 (2009) 036.

[43] S. B. Giddings and A. Maharana, Dynamics of warped compactifications and the shape of the warped landscape, Phys. Rev. D 73, 126003 (2006).

[44] S. P. de Alwis, Constraints on Dbar uplifts, J. High Energy Phys. 11 (2016) 045.

[45] B. Cownden, A. R. Frey, M. C. D. Marsh, and B. Underwood, Dimensional reduction for D3-brane moduli, J. High Energy Phys. 12 (2016) 139.

[46] P. McGuirk, G. Shiu, and F. Ye, Soft branes in supersymmetry-breaking backgrounds, J. High Energy Phys. 07 (2012) 188.

[47] O. DeWolfe and S. B. Giddings, Scales and hierarchies in warped compactifications and brane worlds, Phys. Rev. D 67, 066008 (2003).

[48] D. Baumann, A. Dymarsky, I. R. Klebanov, and L. McAllister, Towards an explicit model of D-brane inflation, J. Cosmol. Astropart. Phys. 01 (2008) 024.

[49] S. Kachru, R. Kallosh, A. D. Linde, J. M. Maldacena, L. P. McAllister, and S. P. Trivedi, Towards inflation in string theory, J. Cosmol. Astropart. Phys. 10 (2003) 013.

[50] J. Moritz, A. Retolaza, and A. Westphal, Toward de Sitter space from ten dimensions, Phys. Rev. D 97, 046010 (2018).

[51] J. Moritz and T. Van Riet, Racing through the swampland: De Sitter uplift vs weak gravity, J. High Energy Phys. 09 (2018) 099.

[52] R. Kallosh, A. Linde, E. McDonough, and M. Scalisi, de Sitter vacua with a nilpotent superfield, Fortschr. Phys. 2018, 1800068 (2018).

[53] J. Moritz, A. Retolaza, and A. Westphal, On uplifts by warped antiD3branes, Fortschr. Phys. 67, 1800098 (2019).

[54] R. Kallosh, A. Linde, E. McDonough, and M. Scalisi, 4D models of de Sitter uplift, Phys. Rev. D 99, 046006 (2019).

[55] F. F. Gautason, V. Van Hemelryck, and T. Van Riet, The tension between 10D supergravity and dS uplifts, Fortschr. Phys. 67, 1800091 (2019).

[56] Y. Hamada, A. Hebecker, G. Shiu, and P. Soler, On brane gaugino condensates in 10d, J. High Energy Phys. 04 (2019) 008.

[57] R. Kallosh, A. Linde, E. McDonough, and M. Scalisi, dS vacua and the swampland, J. High Energy Phys. 03 (2019) 134.

[58] R. Kallosh, Gaugino condensation and geometry of the perfect square, Phys. Rev. D 99, 066003 (2019).

[59] Y. Hamada, A. Hebecker, G. Shiu, and P. Soler, Understanding KKLT from a 10d perspective, J. High Energy Phys. 06 (2019) 019.

[60] F. Carta, J. Moritz, and A. Westphal, Gaugino condensation and small uplifts in KKLT, arXiv:1902.01412.

[61] F. F. Gautason, V. Van Hemelryck, T. Van Riet, and G. Venken, A 10d view on the KKLT AdS vacuum and uplifting, arXiv:1902.01415. 
[62] I. R. Klebanov and M. J. Strassler, Supergravity and a confining gauge theory: Duality cascades and chi SB resolution of naked singularities, J. High Energy Phys. 08 (2000) 052.

[63] O. Aharony, Y.E. Antebi, and M. Berkooz, Open string moduli in KKLT compactifications, Phys. Rev. D 72, 106009 (2005).

[64] S. Gandhi, L. McAllister, and S. Sjors, A toolkit for perturbing flux compactifications, J. High Energy Phys. 12 (2011) 053.

[65] S. Gukov, C. Vafa, and E. Witten, CFT's from Calabi-Yau four folds, Nucl. Phys. B584, 69 (2000).

[66] D. Baumann, A. Dymarsky, S. Kachru, I. R. Klebanov, and L. McAllister, D3-brane potentials from fluxes in AdS/CFT, J. High Energy Phys. 06 (2010) 072.

[67] A. Dymarsky and L. Martucci, D-brane non-perturbative effects and geometric deformations, J. High Energy Phys. 04 (2011) 061.

[68] E. Bergshoeff, R. Kallosh, A.-K. Kashani-Poor, D. Sorokin, and A. Tomasiello, An index for the Dirac operator on D3 branes with background fluxes, J. High Energy Phys. 10 (2005) 102

[69] D. Freedman and A. Van Proeyen, Supergravity (Cambridge University Press, Cambridge, England, 2012).

[70] J. Wess and J. Bagger, Supersymmetry and Supergravity (Princeton University Press, Princeton, NJ, 1992).

[71] M. Rocek, Linearizing the Volkov-Akulov Model, Phys. Rev. Lett. 41, 451 (1978).

[72] U. Lindstrom and M. Rocek, Constrained local superfields, Phys. Rev. D 19, 2300 (1979).

[73] R. Casalbuoni, S. De Curtis, D. Dominici, F. Feruglio, and R. Gatto, Nonlinear realization of supersymmetry algebra from supersymmetric constraint, Phys. Lett. B 220, 569 (1989).

[74] Z. Komargodski and N. Seiberg, From linear SUSY to constrained superfields, J. High Energy Phys. 09 (2009) 066.

[75] S. M. Kuzenko and S. J. Tyler, On the Goldstino actions and their symmetries, J. High Energy Phys. 05 (2011) 055.

[76] F. Farakos and A. Kehagias, Decoupling limits of sGoldstino modes in global and local supersymmetry, Phys. Lett. B 724, 322 (2013).

[77] E. Dudas, S. Ferrara, A. Kehagias, and A. Sagnotti, Properties of nilpotent supergravity, J. High Energy Phys. 09 (2015) 217.

[78] E. A. Bergshoeff, D. Z. Freedman, R. Kallosh, and A. Van Proeyen, Pure de Sitter supergravity, Phys. Rev. D 92, 085040 (2015).

[79] F. Hasegawa and Y. Yamada, Component action of nilpotent multiplet coupled to matter in 4 dimensional $\mathcal{N}=1$ supergravity, J. High Energy Phys. 10 (2015) 106.

[80] S. Ferrara, M. Porrati, and A. Sagnotti, Scale invariant Volkov-Akulov supergravity, Phys. Lett. B 749, 589 (2015).

[81] G. Dall'Agata, E. Dudas, and F. Farakos, On the origin of constrained superfields, J. High Energy Phys. 05 (2016) 041.

[82] S. Ferrara, R. Kallosh, A. Van Proeyen, and T. Wrase, Linear versus non-linear supersymmetry, in general, J. High Energy Phys. 04 (2016) 065.
[83] A. Brignole, F. Feruglio, and F. Zwirner, On the effective interactions of a light gravitino with matter fermions, J. High Energy Phys. 11 (1997) 001.

[84] G. Dall'Agata, S. Ferrara, and F. Zwirner, Minimal scalarless matter-coupled supergravity, Phys. Lett. B 752, 263 (2016).

[85] S. M. Kuzenko, Taking a vector supermultiplet apart: Alternative Fayet-Iliopoulos-type terms, Phys. Lett. B 781, 723 (2018).

[86] I. Antoniadis, A. Chatrabhuti, H. Isono, and R. Knoops, Fayet-Iliopoulos terms in supergravity and D-term inflation, Eur. Phys. J. C 78, 366 (2018).

[87] I. Antoniadis, A. Chatrabhuti, H. Isono, and R. Knoops, The cosmological constant in Supergravity, Eur. Phys. J. C 78, 718 (2018).

[88] Y. Aldabergenov, S. V. Ketov, and R. Knoops, General couplings of a vector multiplet in $N=1$ supergravity with new FI terms, Phys. Lett. B 785, 284 (2018).

[89] Y. Aldabergenov, No-scale supergravity with new FayetIliopoulos term, Phys. Lett. B 795, 366 (2019).

[90] S. M. Kuzenko, Superconformal vector multiplet selfcouplings and generalised Fayet-Iliopoulos terms, Phys. Lett. B 795, 37 (2019).

[91] M. K. Gaillard and B. Zumino, Duality rotations for interacting fields, Nucl. Phys. B193, 221 (1981).

[92] T. Kugo and S. Uehara, $N=1$ superconformal tensor calculus: Multiplets with external Lorentz indices and spinor derivative operators, Prog. Theor. Phys. 73, 235 (1985).

[93] G. Dall'Agata and F. Farakos, Constrained superfields in supergravity, J. High Energy Phys. 02 (2016) 101.

[94] M. Schillo, E. van der Woerd, and T. Wrase, The general de Sitter supergravity component action, Fortschr. Phys. 64, 292 (2016).

[95] Y. Yamada, Construction of higher-derivative supergravity models via superconformal formulation, $\mathrm{Ph}$. D. thesis, Waseda University, 2016.

[96] N. Cribiori, G. Dall'Agata, and F. Farakos, From linear to non-linear SUSY and back again, J. High Energy Phys. 08 (2017) 117.

[97] N. Cribiori, Non-linear realisations in global and local supersymmetry, Ph.D. thesis, Padua University, 2018.

[98] L. Aparicio, F. Quevedo, and R. Valandro, Moduli stabilisation with nilpotent Goldstino: Vacuum structure and SUSY breaking, J. High Energy Phys. 03 (2016) 036.

[99] V. Balasubramanian, P. Berglund, J. P. Conlon, and F. Quevedo, Systematics of moduli stabilisation in CalabiYau flux compactifications, J. High Energy Phys. 03 (2005) 007.

[100] J. P. Conlon, F. Quevedo, and K. Suruliz, Large-volume flux compactifications: Moduli spectrum and D3/D7 soft supersymmetry breaking, J. High Energy Phys. 08 (2005) 007.

[101] R. Kallosh and T. Wrase, dS Supergravity from 10d, Fortschr. Phys. 2018, 1800071 (2018).

[102] H. Jockers and J. Louis, The effective action of D7-branes in $N=1$ Calabi-Yau orientifolds, Nucl. Phys. B705, 167 (2005).

[103] T. W. Grimm and D. V. Lopes, The $N=1$ effective actions of D-branes in type IIA and IIB orientifolds, Nucl. Phys. B855, 639 (2012). 
[104] M. Kerstan and T. Weigand, The effective action of D6branes in $N=1$ type IIA orientifolds, J. High Energy Phys. 06 (2011) 105.

[105] F. Carta, F. Marchesano, W. Staessens, and G. Zoccarato, Open string multi-branched and Kähler potentials, J. High Energy Phys. 09 (2016) 062.

[106] D. Escobar, F. Marchesano, and W. Staessens, Type IIA flux vacua with mobile D6-branes, J. High Energy Phys. 01 (2019) 096.
[107] N. Cribiori, G. Dall'Agata, and F. Farakos, Interactions of N Goldstini in superspace, Phys. Rev. D 94, 065019 (2016).

[108] S. Samuel and J. Wess, A superfield formulation of the nonlinear realization of supersymmetry and its coupling to supergravity, Nucl. Phys. B221, 153 (1983).

[109] F. Farakos, A. Kehagias, and A. Riotto, Liberated $\mathcal{N}=1$ supergravity, J. High Energy Phys. 06 (2018) 011. 